\title{
A posteriori error analysis for two non-overlapping domain decomposition techniques
}

\author{
C. Bernardi*, T. Chacón Rebollo ${ }^{\dagger}$, E. Chacón Vera ${ }^{\ddagger}$, D. Franco Coronil ${ }^{\S}$
}

\begin{abstract}
This paper is devoted to the construction of fast solvers for penalty domain decomposition techniques, based upon a posteriori error analysis. We introduce a penalty non-overlapping domain decomposition method (ddm) motivated by the a posteriori error analysis of the method proposed by Chacón and Chacón in [6]. In the new method a $H_{00}^{1 / 2}(\Gamma)$ penalty term replaces the $L^{2}(\Gamma)$ one in the original method. The number of iterations needed by the new ddm to yield a solution with an error of the same order as the discretization error is remarkably reduced. We develop an a posteriori error analysis that we use to determine an optimal value of the penalty parameter for a given grid, and also to jointly determine an optimal grid and a penalty parameter to reduce the error below a targeted value. Several numerical tests for model problems exhibit the good performances of our approach and provide to a numerical comparison of the two penalty methods.
\end{abstract}

\section{Résumé}

Cet article a pour but la construction de solveurs rapides pour les techniques de décomposition de domaine avec pénalisation et repose sur une analyse a posteriori. Nous introduisons une méthode de décomposition de domaine sans recouvrement, issue de l'analyse a posteriori de la méthode proposée par Chacón et Chacón [6], où une pénalisation de type $H_{00}^{1 / 2}(\Gamma)$ remplace celle de type $L^{2}(\Gamma)$ dans la première méthode. Le nombre d'itérations pour une erreur du même ordre que l'erreur de discrétisation est considérablement réduit. Nous prouvons des estimations d'erreur a posteriori qui permettent d'optimiser le choix du paramètre de pénalisation pour une grille donnée, et aussi lors de l'adaptation de maillage. Plusieurs expériences numériques sur des problèmes académiques montrent les bonnes performances de notre approche et permettent une comparaison numérique des deux méthodes.

${ }^{*}$ Laboratoire Jacques-Louis Lions, C.N.R.S. et Université Pierre et Marie Curie. Boite courrier 187, 4 Place Jussieu, 75252 Paris Cedex 05, France. bernardi@ann.jussieu.fr

†Departamento de Ecuaciones Diferenciales y Análisis Numérico, Universidad de Sevilla. C/ Tarfia, s/n. 41080 Sevilla, Spain. chacon@us.es

${ }_{\ddagger}^{\ddagger}$ Departamento de Ecuaciones Diferenciales y Análisis Numerico, Universidad de Sevilla. C/Tarfia, s/n.41080, Sevilla, Spain. eliseo@us.es

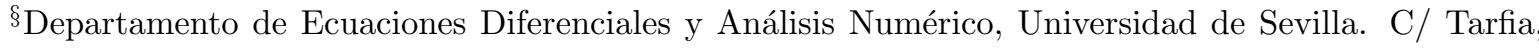
s/n. 41080 Sevilla, Spain. franco@us.es 



\section{Introduction}

This paper is devoted to the construction of fast solvers for penalty domain decomposition techniques, based upon a posteriori error analysis. We introduce a penalty non-overlapping domain decomposition method motivated by the a posteriori error analysis of the method studied by Chacón and Chacón in [6] and [7].

The domain decomposition method (ddm) proposed in [7] enforces the continuity of the variables across the interface between adjacent subdomains through a penalty technique. If we denote this interface by $\Gamma$, a $L^{2}(\Gamma)$ penalty term is added to a convenient variational formulation, with a structure that ensures the continuity of the fluxes across the interface. This method provides fair accurate solutions. In fact, it is proved to be equivalent to a penalty ddm with arbitrarily large overlapping region, analyzed by Lions [11] and Lions and Pironneau [13]. However, it has a rather slow convergence rate. In [7] some solutions to this problem were proposed, particularly the use of general acceleration techniques for sequences, such as Aitken or the Minimal Polynomial Extrapolation methods.

In the present paper we address the problem of finding more specific techniques to improve the convergence rate of the method. At first, we introduce a version of the method in which a $H_{00}^{1 / 2}(\Gamma)$ penalty term replaces the $L^{2}(\Gamma)$ one in the original method of [7]. In this way we force the jump of the unknown to vanish along the interface $\Gamma$ in a stronger sense.

The choice of this new penalty term is suggested by the a posteriori error analysis of the ddm introduced in [7] that we perform in the paper at hand. An improvement is deduced from the a priori error analysis that we develop: If the $L^{2}(\Gamma)$ penalty is replaced by the $H_{00}^{1 / 2}(\Gamma)$ penalty, the number of iterations needed by the ddm to yield a solution with an error of the same order as the discretization error is remarkably reduced. If the discretization error is of order $\mathcal{O}\left(h^{k}\right)$, the $L^{2}(\Gamma)$ penalty requires $\mathcal{O}\left(|\log h| h^{-2 k}\right)$ iterations in order to attain this error, while the $H_{00}^{1 / 2}(\Gamma)$ penalty requires $\mathcal{O}\left(|\log h| h^{-k}\right)$ iterations. This improvement may be explained in an abstract framework: When $H_{00}^{1 / 2}(\Gamma)$ penalty is used, the new ddm may be cast as the penalty of a stable mixed formulation of the Poisson problem. This penalty procedure fits into a general framework studied by Girault and Raviart in [9]. When $L^{2}(\Gamma)$ penalty is used, this mixed formulation is not uniformly stable, due to the lack of an inf-sup condition.

In addition, we develop an a posteriori error analysis that provides independent error indicators for both penalty and discretization errors, and allows to develop strategies to provide further error reductions:

- On one hand, we determine an optimal value for the penalty parameter for a fixed grid that yields a penalty error of the same size as the discretization error, with a minimal computational effort.

- On another hand, we determine both optimal values for the penalty parameter and 
optimal grids that allow to reduce the error below a targeted value, also with a minimal computational effort.

Our results show at first that, indeed, the $H_{00}^{1 / 2}(\Gamma)$ penalty is faster than the $L^{2}(\Gamma)$ one for a fixed grid and a fixed penalty parameter, in good agreement with the a priori error analysis mentioned above. However, surprisingly, a large computational gain is obtained with $L^{2}(\Gamma)$ penalty when an adaptive computation strategy is used. This technique turns out to be more flexible and to need much less computational time per iteration, as it does not require the computation of $H_{00}^{1 / 2}(\Gamma)$ (or even a discrete equivalent) norm.

We refer to [14] for a similar approach (see also [8]). Other types of ddm and the corresponding algorithms are described in [15] and the references therein.

The paper is organized as follows: Section 2 introduces the continuous version of the new ddm. Section 3 performs a joint error analysis of this and the method introduced in [6]. Section 4 characterizes the new ddm as the penalty of a stable mixed method when $H_{00}^{1 / 2}(\Gamma)$ penalty is used. Section 5 is devoted to the discretization by Lagrange finite element spaces of the two methods considered. In Section 6 the a posteriori error analysis of both methods is performed. In Section 7 the improvement of the convergence rate due to the use of $H_{00}^{1 / 2}(\Gamma)$ penalty is analyzed, as an a priori error analysis. Section 8 describes practical strategies for the computation of optimal penalty parameters and grids. In Section 9 we report some numerical tests for the Poisson problem as a model problem in significative geometries, that exhibit the good performances of our approaches. Finally, an Appendix contains the proof of some technical results. 


\section{The penalty problem}

Let $\Omega \subset \mathbb{R}^{d}(d=2,3)$ be a simply connected and bounded domain with a Lipschitzcontinuous boundary $\partial \Omega$. We consider a simple decomposition of $\Omega$ into two non-overlapping subdomains $\Omega_{1}$ and $\Omega_{2}$ and set $\Gamma=\partial \Omega_{1} \cap \partial \Omega_{2}, \Gamma_{i}=\partial \Omega_{i} \cap \partial \Omega$. We assume that all of these boundaries are Lipschitz-continuous $(d-1)$-dimensional manifolds with positive $(d-1)$ dimensional measure. Observe that under these hypotheses, $\Gamma$ may or may not intersect $\partial \Omega$. However, in this case $\Omega$ cannot have a connected boundary, see Figures 1 and 2 .

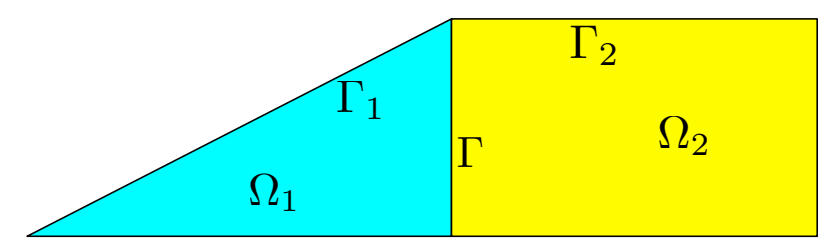

Figure 1: Subdivision of domain $\Omega$ when $\Gamma$ intersects $\partial \Omega$.

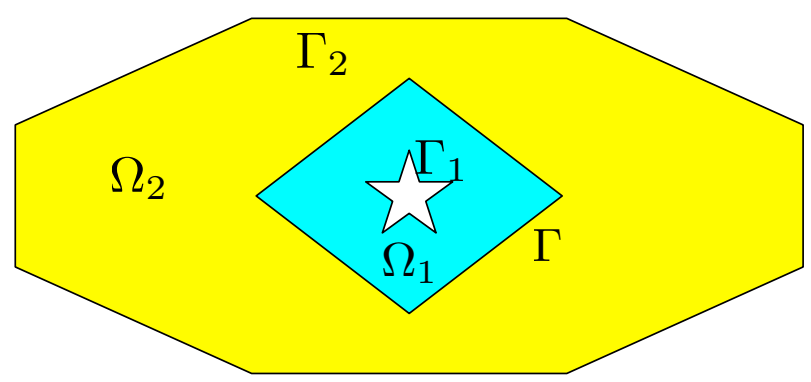

Figure 2: Subdivision of domain $\Omega$ when $\Gamma$ does not intersect $\partial \Omega$.

We denote by $\mathbf{n}_{i j}$ the outward normal vector on $\Gamma$ pointing from $\Omega_{i}$ into $\Omega_{j}, \partial_{\mathbf{n}_{i j}}$ the partial derivative with respect to $\mathbf{n}_{i j}$ and set $\mathbf{n}=\mathbf{n}_{12}$. For a measurable subset $D$ of $\mathbb{R}^{n}$ and two functions $f, g$ such that $f g \in L^{1}(D)$, we denote

$$
(f, g)_{D}=\int_{D} f(x) g(x) d x
$$

We consider the Sobolev spaces

$$
X_{i}=H^{1}\left(\Omega_{i} ; \Gamma_{i}\right)=\left\{v \in H^{1}\left(\Omega_{i}\right) \text { such that } v_{\left.\right|_{\Gamma_{i}}}=0\right\}, \quad i=1,2 ; \quad \mathbf{X}=X_{1} \times X_{2} .
$$


We define for $\mathbf{u}=\left(u_{1}, u_{2}\right), \mathbf{v}=\left(v_{1}, v_{2}\right) \in \mathbf{X}$ the scalar product and norm on $\mathbf{X}$

$$
((\mathbf{u}, \mathbf{v}))_{X}=\sum_{i=1}^{2}\left(\nabla u_{i}, \nabla v_{i}\right)_{\Omega_{i}}, \quad\|\mathbf{u}\|_{X}^{2}=((\mathbf{u}, \mathbf{u}))_{X}
$$

Let us consider the Poisson problem in $\Omega$ :

Given $f \in L^{2}(\Omega)$, find $u \in H_{0}^{1}(\Omega)$ such that

$$
(\nabla u, \nabla v)_{\Omega}=(f, v)_{\Omega}
$$

for all $v \in H_{0}^{1}(\Omega)$. This is a model problem that in despite of its simplicity contains the main difficulties set by the analysis of the ddms that we consider in this paper.

We recall that $H_{00}^{1 / 2}(\Gamma)$ is the subspace of $H^{1 / 2}(\Gamma)$ whose extension by zero to $\partial \Omega_{1}$ (for instance, it could be also to $\left.\partial \Omega_{2}\right)$ belongs to $H^{1 / 2}\left(\partial \Omega_{1}\right)$. An intrinsic scalar product on $H_{00}^{1 / 2}(\Gamma)$ is defined as

$$
\begin{aligned}
{[[w, v]]_{\Gamma}=\int_{\Gamma} w(x) v(x) d x } & +\int_{\Gamma} \int_{\Gamma} \frac{(w(x)-w(y))(v(x)-v(y))}{|x-y|^{d}} d x d y \\
& +\int_{\Gamma} \frac{w(x) v(x)}{d(x, \partial \Gamma)} d x
\end{aligned}
$$

where the first two summands define the $H^{1 / 2}(\Gamma)$ scalar product (see Adams [1], Theorem 7.48). Its expression involves the distance $d(x, \partial \Gamma)$ to the boundary of $\Gamma$. It comes from the restriction to $H_{00}^{1 / 2}(\Gamma)$ of the scalar product in $H^{1 / 2}\left(\partial \Omega_{1}\right)$ for instance. It is given by Lions and Magenes in [12], Chapitre 1, Théorème 11.7.

For brevity, we also denote by $[[\cdot, \cdot]]_{\Gamma}$ the $L^{2}(\Gamma)$ scalar product, and study both the $L^{2}(\Gamma)$ and the $H_{00}^{1 / 2}(\Gamma)$ penalties at the same time with the same notation. We shall distinguish the two cases when this is necessary.

We may now introduce our penalty problem as

$$
\left(P_{\varepsilon}\right) \quad\left\{\begin{array}{l}
\text { Find } \mathbf{u}^{\varepsilon} \in \mathbf{X} \text { such that } \\
\left(\left(\mathbf{u}^{\varepsilon}, \mathbf{v}\right)\right)_{X}+\frac{1}{\varepsilon}\left[\left[u_{1}^{\varepsilon}-u_{2}^{\varepsilon}, v_{1}-v_{2}\right]\right]_{\Gamma}=F(\mathbf{v}) \text { for all } \mathbf{v} \in \mathbf{X} .
\end{array}\right.
$$

where $F$ is the $\|\cdot\|_{X}$-continuous linear form on $\mathbf{X}$ given by

$$
F(\mathbf{v})=\sum_{i=1}^{2}\left(f, v_{i}\right)_{\Omega_{i}}
$$

and $\varepsilon>0$ is a parameter destinated to tend to zero.

This problem has a unique solution for any $\varepsilon>0$ due to Lax-Milgram Lemma. This is also true when the $(d-1)$-dimensional measure of some of the $\Gamma_{i}$ is zero, but we have excluded this case for simplicity. 
When $\Gamma$ does not intersect $\partial \Omega$, then the $H_{00}^{1 / 2}(\Gamma)$ penalty coincides with the $H^{1 / 2}(\Gamma)$ penalty: If $\Gamma$ is a manifold with no boundary, then $H_{00}^{1 / 2}(\Gamma)=H^{1 / 2}(\Gamma)$. However, for brevity we assume that $\Gamma$ intersects $\partial \Omega$ and use $H_{00}^{1 / 2}(\Gamma)$ penalty (or $L^{2}(\Gamma)$ penalty). All our results are also true if $\Gamma$ does not intersect $\partial \Omega$ and $H^{1 / 2}(\Gamma)$ penalty is used.

Remark 1 The original method introduced in [7] only considers a $L^{2}(\Gamma)$ penalty term to enhance the continuity of $\mathbf{u}^{\varepsilon}=\left(u_{1}^{\varepsilon}, u_{2}^{\varepsilon}\right)$ across $\Gamma$. We also consider here a $H_{00}^{1 / 2}(\Gamma)$ penalty term to enhance this continuity in a stronger sense. In both cases, the method may be interpreted as the variational formulation of a coupled system of PDEs with the structure

$$
\left\{\begin{array} { r l r l } 
{ - \Delta u _ { 1 } } & { = f } & { } & { \text { in } \Omega _ { 1 } , } \\
{ u _ { 1 } } & { = 0 } & { \text { on } \Gamma _ { 1 } , } \\
{ \partial _ { \mathbf { n } _ { 1 2 } } u _ { 1 } } & { = \frac { 1 } { \varepsilon } b ( u _ { 1 } - u _ { 2 } ) } & { } & { \text { on } \Gamma , }
\end{array} \quad \left\{\begin{array}{rlrl}
-\Delta u_{2} & =f & & \text { in } \Omega_{2}, \\
u_{2} & =0 & & \text { on } \Gamma_{2}, \\
\partial_{\mathbf{n}_{21}} u_{2} & =\frac{1}{\varepsilon} b\left(u_{2}-u_{1}\right) & & \text { on } \Gamma,
\end{array}\right.\right.
$$

where $b$ is an injective linear bounded boundary operator on $\Gamma$, which reduces to the identity for $L^{2}(\Gamma)$ penalty. So, the method ensures the continuity of the normal fluxes through $\Gamma$ and forces by penalty the continuity of $\mathbf{u}^{\varepsilon}$. Note that, in the case of $H_{00}^{1 / 2}(\Gamma)$ penalty, this is rather different from a Dirichlet to Neumann or Neumann to Neumann algorithm. 


\section{Penalty error analysis}

Our first result is a generalization of the penalty error estimates of [7]. To state it we denote by $\|\cdot\|_{\Gamma}$ the norm associated with the $[[\cdot, \cdot]]_{\Gamma}$ scalar product, and by $\|\cdot\|_{*, \Gamma}$ its dual norm.

We use the notation

$$
((\mathbf{u}, \mathbf{v}))_{\varepsilon}=((\mathbf{u}, \mathbf{v}))_{X}+\frac{1}{\varepsilon}\left[\left[u_{1}-u_{2}, v_{1}-v_{2}\right]\right]_{\Gamma}, \quad \forall \mathbf{u}, \mathbf{v} \in \mathbf{X}
$$

Theorem 1 Assume that one of the following assumptions hold:

- Either the solution $u$ of (1) satisfies $\partial_{\mathbf{n}} u \in L^{2}(\Gamma)$ when $L^{2}(\Gamma)$ penalty is used,

- Or $f$ belongs to $L^{2}(\Omega)$ when $H_{00}^{1 / 2}(\Gamma)$ penalty is used.

Then, for all $\varepsilon>0$ the following estimates hold

$$
\left\|u_{1}^{\varepsilon}-u_{2}^{\varepsilon}\right\|_{\Gamma} \leq\left\|\partial_{\mathbf{n}} u\right\|_{*, \Gamma} \varepsilon, \quad \sum_{i=1}^{2}\left|u-u_{i}^{\varepsilon}\right|_{1, \Omega_{i}} \leq\left\|\partial_{\mathbf{n}} u\right\|_{*, \Gamma} \sqrt{\varepsilon}
$$

Proof: The case of $L^{2}(\Gamma)$ penalty has been proved in [7] (Theorem 1), we include it in the statement of the present theorem for completeness. We prove $(3)$ in the case of $H_{00}^{1 / 2}(\Gamma)$ penalty.

For the solution $u$ of problem (1) we write $\mathbf{u}=\left(u_{\left.\right|_{\Omega_{1}}}, u_{\left.\right|_{\Omega_{2}}}\right) \in \mathbf{X}$. Consider the consistency error $\mathcal{C} \in \mathbf{X}^{\prime}$ defined by

$$
\langle\mathcal{C}, \mathbf{v}\rangle=((\mathbf{u}, \mathbf{v}))_{\varepsilon}-F(\mathbf{v}), \forall \mathbf{v}=\left(v_{1}, v_{2}\right) \in \mathbf{X} .
$$

Then the following property holds

$$
\left(\left(\mathbf{u}-\mathbf{u}_{\varepsilon}, \mathbf{v}\right)\right)_{\varepsilon}=\langle\mathcal{C}, \mathbf{v}\rangle, \forall \mathbf{v}=\left(v_{1}, v_{2}\right) \in \mathbf{X} .
$$

To estimate the consistency error, observe that as $\Delta u \in L^{2}\left(\Omega_{i}\right)$, then $\partial_{\mathbf{n}_{12}} u \in\left(H_{00}^{1 / 2}(\Gamma)\right)^{\prime}$ due to the relation

$$
<\partial_{\mathbf{n}_{i j}} u, v_{i}>_{\Gamma}=\left(\nabla u, \nabla v_{i}\right)_{\Omega_{i}}+\left(\Delta u, v_{i}\right)_{\Omega_{i}}, \forall v_{i} \in X_{i} .
$$

Thus, we may integrate by parts in the expression of $\mathcal{C}$ to obtain

$$
\langle\mathcal{C}, \mathbf{v}\rangle=<\partial_{\mathbf{n}} u, v_{1}-v_{2}>_{\Gamma} .
$$

We may now use the error equation (5) with $\mathbf{v}=\mathbf{u}-\mathbf{u}_{\varepsilon}$ to obtain

$$
\sum_{i=1}^{2}\left|u-u_{i}^{\varepsilon}\right|_{1, \Omega_{i}}^{2}+\frac{1}{\varepsilon}\left\|u_{1}^{\varepsilon}-u_{2}^{\varepsilon}\right\|_{H_{00}^{1 / 2}(\Gamma)}^{2}=<\partial_{\mathbf{n}} u, u_{2}^{\varepsilon}-u_{1}^{\varepsilon}>_{\Gamma} \leq\left\|\partial_{\mathbf{n}} u\right\|_{\left(H_{00}^{1 / 2}(\Gamma)\right)^{\prime}}\left\|u_{1}^{\varepsilon}-u_{2}^{\varepsilon}\right\|_{H_{00}^{1 / 2}(\Gamma)} .
$$


By Young's inequality,

$$
\sum_{i=1}^{2}\left|u-u_{i}^{\varepsilon}\right|_{1, \Omega_{i}}^{2}+\frac{1}{\varepsilon}\left\|u_{1}^{\varepsilon}-\left.u_{2}^{\varepsilon}\right|_{H_{00}^{1 / 2}(\Gamma)} ^{2} \leq \frac{\varepsilon}{2}\right\| \partial_{\mathbf{n}} u\left\|_{\left(H_{00}^{1 / 2}(\Gamma)\right)^{\prime}}^{2}+\frac{1}{2 \varepsilon}\right\| u_{1}^{\varepsilon}-u_{2}^{\varepsilon} \|_{H_{00}^{1 / 2}(\Gamma)}^{2}
$$

This yields estimates (3).

Q.E.D.

We next introduce a continuous penalty error indicator (and refer to [14], Thm 3.1, for a similar indicator):

$$
\eta^{P}=\left\|u_{1}^{\varepsilon}-u_{2}^{\varepsilon}\right\|_{H_{00}^{1 / 2}(\Gamma)} .
$$

This is an optimal indicator, as it satisfies

Theorem 2 There exist two constants $C>0$ and $C^{\prime}>0$ independent of $\varepsilon$ such that

$$
C \eta^{P} \leq \sum_{i=1}^{2}\left|u-u_{i}^{\varepsilon}\right|_{1, \Omega_{i}} \leq C^{\prime} \eta^{P}, i=1,2 .
$$

Proof: The first estimate in (8) is obtained by

$$
\left\|u_{1}^{\varepsilon}-u_{2}^{\varepsilon}\right\|_{H_{00}^{1 / 2}(\Gamma)} \leq \sum_{i=1}^{2}\left\|u-u_{i}^{\varepsilon}\right\|_{H_{00}^{1 / 2}(\Gamma)} \leq C_{1} \sum_{i=1}^{2}\left\|u-u_{i}^{\varepsilon}\right\|_{H^{1 / 2}\left(\partial \Omega_{i}\right)},
$$

whence

$$
\left\|u_{1}^{\varepsilon}-u_{2}^{\varepsilon}\right\|_{H_{00}^{1 / 2}(\Gamma)} \leq C_{2} \sum_{i=1}^{2}\left\|u-u_{i}^{\varepsilon}\right\|_{1, \Omega_{i}} .
$$

This inequality stems from the usual characterization of the $H_{00}^{1 / 2}(\Gamma)$ norm (equivalent to the intrinsic one given by (2), Cf. Adams [1], Theorem 7.53) defined as

$$
\inf _{w \in X_{i}, w_{\Gamma}=\varphi}\|w\|_{1, \Omega_{i}}
$$

To prove the second estimate in (8), let us introduce the subspace of $\mathbf{X}$,

$$
\widetilde{\mathbf{X}}=\left\{\tilde{\mathbf{v}}=\left(\tilde{v}_{1}, \tilde{v}_{2}\right) \in \mathbf{X} \text {, such that } \tilde{v}_{1_{\left.\right|_{\Gamma}}}=\tilde{v}_{2_{\left.\right|_{\Gamma}}}\right\}
$$

which is algebraically isomorphic to $H_{0}^{1}(\Omega)$. Observe that

$$
\left(\left(\mathbf{u}-\mathbf{u}^{\varepsilon}, \tilde{\mathbf{v}}\right)\right)_{X}=0, \forall \tilde{\mathbf{v}} \in \widetilde{\mathbf{X}}
$$


There exists a lifting $w_{1}^{\varepsilon} \in X_{1}$ of $\left(u_{1}^{\varepsilon}-u_{2}^{\varepsilon}\right)_{\Gamma}$ satisfying

$$
\left|w_{1}^{\varepsilon}\right|_{1, \Omega_{1}} \leq C_{3}\left\|u_{1}^{\varepsilon}-u_{2}^{\varepsilon}\right\|_{H_{00}^{1 / 2}(\Gamma)} .
$$

for some constant $C_{3}$ independent of $\varepsilon$. For instance, this lifting is obtained as the solution of the boundary value problem

$$
-\Delta w_{1}^{\varepsilon}=0 \text { in } \Omega_{1}, \quad w_{1}^{\varepsilon}=0 \text { on } \Gamma_{1}, \quad w_{1}^{\varepsilon}=u_{1}^{\varepsilon}-u_{2}^{\varepsilon} \text { on } \Gamma .
$$

Define the function $\mathbf{w}^{\varepsilon}=\left(w_{1}^{\varepsilon}, 0\right) \in \mathbf{X}$. Then, taking $\tilde{\mathbf{v}}=\mathbf{u}-\mathbf{u}^{\varepsilon}+\mathbf{w}^{\varepsilon} \in \widetilde{\mathbf{X}}$ in (9), we have

$$
\left\|\mathbf{u}-\mathbf{u}^{\varepsilon}\right\|_{X} \leq\left\|\mathbf{w}^{\varepsilon}\right\|_{X}, \text { and so } \sum_{i=1}^{2}\left|u-u_{i}^{\varepsilon}\right|_{1, \Omega_{i}} \leq \sqrt{2}\left|w_{1}^{\varepsilon}\right|_{1, \Omega_{i}} \leq C_{4} \eta^{P}
$$

Q.E.D.

Observe that the a posteriori error estimator $\eta^{P}$ coincides with the $H_{00}^{1 / 2}(\Gamma)$ penalty term. In this case, the error estimate for $\left|u-u_{i}^{\varepsilon}\right|_{1, \Omega_{i}}$ stated in Theorem 1 is improved by combining the second estimate in (8) with the first estimate in (3) :

Corollary 3 Assume that $H_{00}^{1 / 2}(\Gamma)$ penalty is used and that $f$ belongs to $L^{2}(\Omega)$.

Then, for all $\varepsilon>0$ the following estimate holds

$$
\sum_{i=1}^{2}\left|u-u_{i}^{\varepsilon}\right|_{1, \Omega_{i}} \leq C\left\|\partial_{\mathbf{n}} u\right\|_{H_{00}^{1 / 2}(\Gamma)^{\prime}} \varepsilon
$$

for some constant $C$ independent of $\varepsilon$.

This suggests to replace the $L^{2}(\Gamma)$ penalty term by the $H_{00}^{1 / 2}(\Gamma)$ penalty term to improve the accuracy of our ddm. From the practical point of view this sets the problem of the computation of the non-local $H_{00}^{1 / 2}(\Gamma)$ norm and scalar products. However, these can be approximated numerically with low cost. We show the good performances of such an approach in our numerical results. 


\section{Casting as a penalty mixed problem}

In the case of $H_{00}^{1 / 2}(\Gamma)$ penalty, Problem $\left(P_{\varepsilon}\right)$ may be cast as a penalty mixed problem, that fits into the general framework of approximation of mixed problems by penalty analysed in Chapter I, Section 4.3 of Girault and Raviart [9]. We also refer to [2] for the introduction of a Lagrangian multiplier to handle the matching conditions in the ddm. To do this, let us denote by $M$ the space $H_{00}^{1 / 2}(\Gamma)$, and define the bilinear bounded form

$$
b(\cdot, \cdot): \mathbf{X} \times M \mapsto \mathbb{R} \quad \text { by } \quad b(\mathbf{v}, \lambda)=\left[\left[v_{1}-v_{2}, \lambda\right]\right]_{\Gamma} .
$$

We re-formulate the Poisson problem (1) as the following mixed problem:

Find a pair $\left(\tilde{\mathbf{u}}=\left(\tilde{u}_{1}, \tilde{u}_{2}\right), \lambda\right) \in \mathbf{X} \times M$ such that

$$
\left(P_{M}\right) \quad\left\{\begin{array}{rlrl}
((\tilde{\mathbf{u}}, \mathbf{v}))_{X}+b(\mathbf{v}, \lambda) & =F(\mathbf{v}), & & \forall \mathbf{v} \in \mathbf{X} \\
b(\tilde{\mathbf{u}}, \mu) & =0, & \forall \mu \in M
\end{array}\right.
$$

It can be readily checked that the form $b(\cdot, \cdot)$ satisfies an inf-sup condition: There exists a constant $\beta>0$ such that

$$
\beta\|\mu\|_{M} \leq \sup _{\mathbf{v} \in \mathbf{X}} \frac{b(\mathbf{v}, \mu)}{\|\mathbf{v}\|_{X}}, \quad \forall \mu \in M .
$$

Thus, problem $\left(P_{M}\right)$ admits a unique solution $(\tilde{\mathbf{u}}, \lambda)$. From the second equation in (11), $\tilde{u}_{1}=\tilde{u}_{2}$ on $\Gamma$. Then, the solution of the Poisson problem is recovered from that of $\left(P_{M}\right)$ by

$$
u(x)=\left\{\begin{array}{lll}
\tilde{u}_{1}(x) & \text { if } & x \in \Omega_{1}, \\
\tilde{u}_{2}(x) & \text { if } & x \in \Omega_{2} .
\end{array}\right.
$$

Conversely, if we define $\tilde{u}_{i}=u_{\left.\right|_{\Omega_{i}}}$, then the second equation in (11) holds and the multiplier $\lambda$ is obtained from the first equation in (11) thanks to the inf-sup condition (12).

Let us now consider the scalar product in $H_{00}^{1 / 2}(\Gamma)$,

$$
c(\lambda, \mu)=[[\lambda, \mu]]_{\Gamma}
$$

and the penalty problem

Find a pair $\left(\tilde{\mathbf{u}}^{\varepsilon}, \lambda^{\varepsilon}\right) \in \mathbf{X} \times M$ such that

$$
\left(P_{M}^{\varepsilon}\right) \quad\left\{\begin{array}{rlrl}
\left(\left(\tilde{\mathbf{u}}^{\varepsilon}, \mathbf{v}\right)\right)_{X}+b\left(\mathbf{v}, \lambda^{\varepsilon}\right) & =F(\mathbf{v}), & & \forall \mathbf{v} \in \mathbf{X} \\
-\varepsilon c\left(\lambda^{\varepsilon}, \mu\right)+b\left(\tilde{\mathbf{u}}^{\varepsilon}, \mu\right) & =0, & \forall \mu \in M
\end{array}\right.
$$

This problem is equivalent to $\left(P_{\varepsilon}\right)$. Indeed, from the second equation in (13) we obtain

$$
\lambda^{\varepsilon}=\frac{1}{\varepsilon}\left(\tilde{u}_{1}^{\varepsilon}-\tilde{u}_{2}^{\varepsilon}\right) .
$$


Inserting this equality in the first equation of $(13), \tilde{\mathbf{u}}^{\varepsilon}$ turns out to verify exactly $\left(P_{\varepsilon}\right)$.

Problem (13) is studied in an abstract framework in Chapter I, Section 4.3 of [9]. Its well-posedness is proved, so as the error estimate

$$
\left\|\tilde{\mathbf{u}}^{\varepsilon}-\tilde{\mathbf{u}}\right\|_{X}+\left\|\lambda^{\varepsilon}-\lambda\right\|_{M} \leq K \varepsilon\|F\|_{X^{\prime}}
$$

where $K$ is a constant depending on $\Omega, \Gamma, d$ and $\beta$. This estimate is similar to the ones that we have obtained in Theorem 1 and Corollary 3.

Observe that the $L^{2}(\Gamma)$ penalty does not fit into this framework, as an inf-sup condition (12) does not hold. Indeed, in this case, the quantity

$$
\sup _{\mathbf{v} \in \mathbf{X}} \frac{b(\mathbf{v}, \mu)}{\|\mathbf{v}\|_{X}},
$$

is a norm equivalent to the $\left(H_{00}^{1 / 2}(\Gamma)\right)^{\prime}$ norm, and not to the $L^{2}(\Gamma)$ norm. 


\section{Discretization of the penalty problem}

To discretize $\left(P_{\varepsilon}\right)$ let us assume that the domains $\Omega, \Omega_{1}$ and $\Omega_{2}$ are polygonal or polyhedral. Consider a regular family of triangulations $\left\{\mathcal{T}_{i h}\right\}_{\{h>0\}}$ of each $\bar{\Omega}_{i}$ formed by polygons $(d=2)$ or polyhedra $(d=3)$ such that $\Gamma$ is the union of whole faces or sides of elements $K$ in each $\mathcal{T}_{i h}$. As usual, $h>0$ denotes the largest diameter of the elements of $\mathcal{T}_{h}=\mathcal{T}_{1 h} \cup \mathcal{T}_{2 h}$.

We assume that $\mathcal{T}_{1 h}$ and $\mathcal{T}_{2 h}$ have the same trace sets on $\Gamma$; i.e., the trace sets

$$
\partial_{\Gamma} \mathcal{T}_{i h}=\left\{e \subset \mathbb{R}^{d} \text { such that } e=\partial K \cap \bar{\Gamma} \text { for some } K \in \mathcal{T}_{i h}\right\} ; i=1,2
$$

of $\mathcal{T}_{1 h}$ and $\mathcal{T}_{2 h}$ are equal to a same set, that we denote by $\mathcal{E}_{h}^{\Gamma}$. Then, $\mathcal{T}_{h}$ is a triangulation of $\Omega$, and the family $\left\{\mathcal{T}_{h}\right\}_{\{h>0\}}$ is regular.

Next we consider a family of finite element subspaces $X_{i h}$ of $X_{i}(i=1,2)$, built on the grids $\mathcal{T}_{i h}$. Note that the two spaces $X_{1 h}$ and $X_{2 h}$ may be constructed with piecewise polynomials of different degrees, or different finite elements.

We set $\mathbf{X}_{h}=X_{1 h} \times X_{2 h}$. We define the spaces

$$
\tilde{\mathbf{X}}_{h}=\left\{\tilde{\mathbf{v}}_{h}=\left(\tilde{v}_{1 h}, \tilde{v}_{2 h}\right) \in \mathbf{X}_{h} \text {, such that } \tilde{v}_{1 h_{\mid \Gamma}}=\tilde{v}_{2 h_{\mid \Gamma}}\right\} \text {, and }
$$

$$
V_{h}=\left\{v_{h}: \bar{\Omega} \mapsto \mathbb{R}^{d} \text { such that } v_{\left.h\right|_{\Omega_{1}}}=\tilde{v}_{1 h} \text { and } v_{\left.h\right|_{\Omega_{2}}}=\tilde{v}_{2 h} \text { for some }\left(\tilde{v}_{1 h}, \tilde{v}_{2 h}\right) \in \tilde{\mathbf{X}}_{h}\right\} \text {. }
$$

Then, $V_{h}$ is a finite element subspace of $H_{0}^{1}(\Omega)$ built on the triangulation $\mathcal{T}_{h}$. It is not reduced to $\{0\}$ in the situations that we take into consideration.

The structure of these finite element spaces allows compatible discretizations of our penalty ddm, in the sense that a discrete orthogonality property (9) holds. We consider the standard finite element Galerkin approximation $u_{h} \in V_{h}$ of $u$, satisfying

$$
\left(P_{h}\right) \quad\left\{\begin{array}{l}
\text { Find } u_{h} \in V_{h} \text { such that } \\
\left(\nabla u_{h}, \nabla v_{h}\right)_{\Omega}=\left(f, v_{h}\right)_{\Omega} \text { for all } v_{h} \in V_{h} .
\end{array}\right.
$$

Our penalty discrete problem is the standard finite element Galerkin approximation $\mathbf{u}_{h}^{\varepsilon}=\left(u_{1 h}^{\varepsilon}, u_{2 h}^{\varepsilon}\right) \in \mathbf{X}_{h}$ of $\mathbf{u}^{\varepsilon}$, solution of

$$
\left(P_{\varepsilon, h}\right) \quad\left\{\begin{array}{l}
\text { Find } \mathbf{u}_{h}^{\varepsilon} \in \mathbf{X}_{h} \text { such that } \\
\left(\left(\mathbf{u}_{h}^{\varepsilon}, \mathbf{v}_{h}\right)\right)_{\varepsilon}=F\left(\mathbf{v}_{h}\right) \text { for all } \mathbf{v}_{h} \in \mathbf{X}_{h} .
\end{array}\right.
$$

This problem admits a unique solution for any $\varepsilon>0$.

We consider the parallel technique by Schwarz successive approximations introduced in [7] to solve $\left(P_{\varepsilon, h}\right)$ :

For $n=0,1,2, \ldots, u_{1}^{n+1}=u_{1 h}^{n+1, \varepsilon} \in X_{1 h}$ and $u_{2}^{n+1}=u_{2 h}^{n+1, \varepsilon} \in X_{2 h}$ are computed from $u_{1 h}^{n}$ and $u_{1 h}^{n}$ by solving (we drop the exponent $\varepsilon$ and index $h$ for simplicity),

$$
\left(P_{\varepsilon, h}^{n}\right)\left\{\begin{array}{l}
\left(\nabla u_{1}^{n+1}, \nabla v_{1 h}\right)_{\Omega_{1}}+\frac{1}{\varepsilon}\left[\left[u_{1}^{n+1}-u_{2}^{n}, v_{1 h}\right]\right]_{\Gamma}=\left(f, v_{1 h}\right)_{\Omega_{1}}, \quad \forall v_{1 h} \in X_{1 h}, \\
\left(\nabla u_{2}^{n+1}, \nabla v_{2 h}\right)_{\Omega_{2}}+\frac{1}{\varepsilon}\left[\left[u_{2}^{n+1}-u_{1}^{n}, v_{2 h}\right]\right]_{\Gamma}=\left(f, v_{2 h}\right)_{\Omega_{2}}, \quad \forall v_{2 h} \in X_{2 h} .
\end{array}\right.
$$


For $k \geq 1$ we assume that $u_{h}$ is a finite element approximation of degree $k$ of $u$, in the sense that when $u \in H^{k+1}(\Omega) \cap H_{0}^{1}(\Omega)$ the error committed with $u_{h}$ belongs to $V_{h}$ is $\mathcal{O}\left(h^{k}\right) ;$ specifically

$$
\left|u_{h}-u\right|_{1, \Omega} \leq C_{0} h^{k}|u|_{k+1, \Omega}
$$

for some constant $C_{0}>0$.

We also assume that the spaces $X_{i h}$ verify the approximation estimates

$$
\begin{aligned}
\forall v_{i} \in X_{i}, \exists v_{i h} \in X_{i h} \text { s. t. }\left\|v_{i}-v_{i h}\right\|_{\alpha, \Omega_{i}} & \leq C_{0} h^{1-\alpha}\left|v_{i}\right|_{1, \Omega_{i}}, 0 \leq \alpha \leq 1, \\
& \text { and } \\
\| v_{i}-\left.v_{i h}\right|_{\alpha, \Gamma} & \leq C_{0} h^{1 / 2-\alpha}\left|v_{i}\right|_{1, \Omega_{i}}, 0 \leq \alpha \leq 1 / 2 .
\end{aligned}
$$

Sufficient conditions that guarantee that rather general families of affine equivalent finite element spaces satisfy these properties are given in Bernardi et al. [4], Chapitre IX (Cf. Corollaire IX.3.9 and Théorème IX.3.10 for the proofs). In particular, these conditions are verified by the Lagrange finite element spaces constructed with triangles $(d=2)$ or tetrahedra $(d=3)$, as

$$
X_{i h}^{*}=\left\{w_{h} \in H^{1}\left(\Omega_{i}\right) ; w_{h_{\left.\right|_{K}}} \in P_{k_{i}}(K), \forall K \in \mathcal{T}_{i h}, w_{h_{\Gamma_{i}}}=0\right\},
$$

where $P_{k_{i}}(K)$ denotes the space of restrictions to $K$ of polynomials with $d$ variables and total degre $\leq k_{i}$ for positive integers $k_{i}$.

Note that if $k_{1} \neq k_{2}$, say $k_{1}>k_{2}$, then the first component $v_{1 h} \in X_{1 h}^{*}$ of a pair $\left(v_{1 h}, v_{2 h}\right) \in \widetilde{\mathbf{X}}_{h}$ must degenerate to polynomials of $P_{k_{2}}(e)$ on the sides or faces $e \in \mathcal{E}_{h}^{\Gamma}$ when the $X_{i h}$ are equal to the $X_{i h}^{*}$. 


\section{A posteriori error analysis}

From now on, we assume that each of the spaces $X_{i h}$ contains the space $X_{i h}^{*}$ defined in (18), and that its trace space on $\Gamma$ is

$$
W_{i h}=\left\{v_{h} \in H_{0}^{1}(\Gamma): v_{\left.h\right|_{e}} \in P_{k_{i}}(e), \forall e \in \mathcal{E}_{h}^{\Gamma}\right\} .
$$

To introduce our error indicators, for each $K \in \mathcal{T}_{i h}, i=1$, 2 , let us denote by $\mathcal{E}_{K}$ the set of edges $(d=2)$ or faces $(d=3)$ of $K$ that are not contained in $\Gamma_{i}$. Let us also consider a piecewise polynomial approximation $f_{h}$ of $f$ on the triangulation $\mathcal{T}_{h}$. We introduce

- a family of discretization error indicators: For $i=1,2$ and $K \in \mathcal{T}_{i h}$,

$$
\eta_{i}^{K}=h_{K}\left\|f_{h}+\Delta u_{i h}^{\varepsilon}\right\|_{L^{2}(K)}+\sum_{e \in \mathcal{E}_{K}} h_{e}^{1 / 2}\left\|\left[\partial_{n_{K}} u_{i h}^{\varepsilon}\right]\right\|_{L^{2}(e)},
$$

where

- $h_{K}$ and $h_{e}$ stand for for the diameters of $K$ and $e$, respectively,

- $n_{K}$ stands for the unit outward normal to $\partial K$, and

- $\left[\partial_{n_{K}} u_{i h}^{\varepsilon}\right]$ stands for the jump of $\partial_{n_{K}} u_{i h}^{\varepsilon}$ across $e$ if $e$ is not included in $\Gamma$, and for $\partial_{n_{K}} u_{1 h}^{\varepsilon}-\partial_{n_{K}} u_{2 h}^{\varepsilon}$ if $e$ is included in $\Gamma$.

- a penalty error indicator:

$$
\eta_{h}^{P}=\left\|u_{1 h}^{\varepsilon}-u_{2 h}^{\varepsilon}\right\|_{H_{00}^{1 / 2}(\Gamma)} .
$$

The penalty error is estimated by the following discrete version of Theorem 2 :

Theorem 4 There exist two constants $C>0$ and $C^{\prime}>0$ independent of $\varepsilon$ and $h$ such that

$$
C \eta_{h}^{P} \leq \sum_{i=1}^{2}\left|u_{h}-u_{i h}^{\varepsilon}\right|_{1, \Omega_{i}} \leq C^{\prime} \eta_{h}^{P}, i=1,2 .
$$

Proof: It is parallel to that of Theorem 2. The first inequality is proved in the same way.

To prove the second inequality, we start from the discrete orthogonality property

$$
\left(\left(\mathbf{u}_{h}-\mathbf{u}_{h}^{\varepsilon}, \tilde{\mathbf{v}}_{h}\right)\right)_{X}=0, \forall \tilde{\mathbf{v}}_{h} \in \widetilde{\mathbf{X}}_{h}, \text { with } \mathbf{u}_{h}=\left(u_{1 h}, u_{2 h}\right), \mathbf{u}_{h}^{\varepsilon}=\left(u_{1 h}^{\varepsilon}, u_{2 h}^{\varepsilon}\right) .
$$

Denote by $\varphi_{h}$ the extension by zero of $\left(u_{1 h}^{\varepsilon}-u_{2 h}^{\varepsilon}\right)_{\left.\right|_{\Gamma}}$ to $\partial \Omega_{1}$. Since $\left(u_{1 h}^{\varepsilon}-u_{2 h}^{\varepsilon}\right)_{\left.\right|_{\Gamma}}$ vanishes on $\partial \Gamma$, assuming for instance $k_{1} \geq k_{2}$, then $\varphi_{h}$ belongs to the trace space on $\partial \Omega_{1}$ of $X_{1 h}$. Thus, following Bernardi et al. [4] (Théorème IX.4.1), there exists a lifting $w_{1 h}^{\varepsilon} \in X_{1 h}$ of $\varphi_{h}$ that satisfies

$$
\left|w_{1 h}^{\varepsilon}\right|_{1, \Omega_{1}} \leq C_{1}\left\|\varphi_{h}\right\|_{H^{1 / 2}\left(\partial \Omega_{1}\right)}
$$


for some constant $C_{1}$ independent of $\varepsilon$. As

$$
\left\|\varphi_{h}\right\|_{H^{1 / 2}\left(\partial \Omega_{1}\right)} \leq C_{2}\left\|u_{1 h}^{\varepsilon}-u_{2 h}^{\varepsilon}\right\|_{H_{00}^{1 / 2}(\Gamma)}, \text { then }\left|w_{1 h}^{\varepsilon}\right|_{1, \Omega_{1}} \leq C_{3}\left\|u_{1 h}^{\varepsilon}-u_{2 h}^{\varepsilon}\right\|_{H_{00}^{1 / 2}(\Gamma)} \text {. }
$$

Define the function $\mathbf{w}_{h}^{\varepsilon}=\left(w_{1 h}^{\varepsilon}, 0\right) \in \mathbf{X}_{h}$. Then, it is enough to take $\tilde{\mathbf{v}}_{h}=\mathbf{u}_{h}-\mathbf{u}_{h}^{\varepsilon}+\mathbf{w}_{h}^{\varepsilon} \in \widetilde{\mathbf{X}}_{h}$ in (21) and repeat the remaining arguments of the end of proof of Theorem 2.

Q.E.D.

We may now state our first a posteriori error analysis result:

Theorem 5 The solution of the discrete penalty problem $\left(P_{h, \varepsilon}\right)$ satisfies the following a posteriori error estimate

$$
\begin{aligned}
\sum_{i=1}^{2}\left|u-u_{i h}^{\varepsilon}\right|_{1, \Omega_{i}} & \leq C\left\{\left(1+\frac{h^{\gamma}}{\varepsilon}\right) \eta_{h}^{P}\right. \\
& \left.+\left(\sum_{i=1}^{2} \sum_{K \in \mathcal{T}_{i h}}\left(\eta_{i}^{K}\right)^{2}+h_{K}^{2}\left\|f-f_{h}\right\|_{L^{2}(K)}^{2}\right)^{1 / 2}\right\},
\end{aligned}
$$

where $\gamma= \begin{cases}1 / 2 & \text { if } L^{2}(\Gamma) \text { penalty is used, } \\ 0 & \text { if } H_{00}^{1 / 2}(\Gamma) \text { penalty is used. }\end{cases}$

Proof. We start from the triangle inequality

$$
\sum_{i=1}^{2}\left|u-u_{i h}^{\varepsilon}\right|_{1, \Omega_{i}} \leq \sum_{i=1}^{2}\left|u-u_{i}^{\varepsilon}\right|_{1, \Omega_{i}}+\sum_{i=1}^{2}\left|u_{i}^{\varepsilon}-u_{i h}^{\varepsilon}\right|_{1, \Omega_{i}}
$$

To estimate the first term in the right-hand side of (23), we use (8):

$$
\sum_{i=1}^{2}\left|u-u_{i}^{\varepsilon}\right|_{1, \Omega_{i}} \leq C_{1}\left\|u_{1}^{\varepsilon}-u_{2}^{\varepsilon}\right\|_{H_{00}^{1 / 2}(\Gamma)} \leq C_{1}\left(\left\|u_{1 h}^{\varepsilon}-u_{2 h}^{\varepsilon}\right\|_{H_{00}^{1 / 2}(\Gamma)}+\sum_{i=1}^{2}\left\|u_{i}^{\varepsilon}-u_{i h}^{\varepsilon}\right\|_{H_{00}^{1 / 2}(\Gamma)}\right) .
$$

Combining this estimate with (23) we obtain

$$
\sum_{i=1}^{2}\left|u-u_{i h}^{\varepsilon}\right|_{1, \Omega_{i}} \leq C_{2}\left(\eta_{h}^{P}+\sum_{i=1}^{2}\left|u_{i}^{\varepsilon}-u_{i h}^{\varepsilon}\right|_{1, \Omega_{i}}\right)
$$

for some constant $C_{2} \geq 0$ independent of $\varepsilon$ and $h$.

To estimate the last term in the right-hand side of this inequality, we use the problems $\left(P_{\varepsilon}\right)$ and $\left(P_{\varepsilon, h}\right)$ to deduce the residual equation:

$$
\left(\left(\mathbf{u}^{\varepsilon}-\mathbf{u}_{h}^{\varepsilon}, \mathbf{v}\right)\right)_{\varepsilon}=F(\mathbf{v})-\left(\left(\mathbf{u}_{h}^{\varepsilon}, \mathbf{v}\right)\right)_{\varepsilon}=F\left(\mathbf{v}-\mathbf{v}_{h}\right)-\left(\left(\mathbf{u}_{h}^{\varepsilon}, \mathbf{v}-\mathbf{v}_{h}\right)\right)_{\varepsilon}, \forall \mathbf{v} \in \mathbf{X}, \forall \mathbf{v}_{h} \in \mathbf{X}_{h} .
$$


Then,

$$
\left(\left(\mathbf{u}^{\varepsilon}-\mathbf{u}_{h}^{\varepsilon}, \mathbf{v}\right)\right)_{\varepsilon}=\sum_{i=1}^{2}\left(f_{h}, v_{i}-v_{i h}\right)_{\Omega_{i}}-\left(\left(\mathbf{u}_{h}^{\varepsilon}, \mathbf{v}-\mathbf{v}_{h}\right)\right)_{\varepsilon}+\sum_{i=1}^{2}\left(f-f_{h}, v_{i}-v_{i h}\right)_{\Omega_{i}} .
$$

Whence,

$$
\begin{aligned}
\left(\left(\mathbf{u}^{\varepsilon}-\mathbf{u}_{h}^{\varepsilon}, \mathbf{v}\right)\right)_{\varepsilon} & =\sum_{i=1}^{2} \sum_{K \in \mathcal{T}_{i h}}\left(\int_{K}\left(f_{h}+\Delta u_{i h}^{\varepsilon}\right)\left(v_{i}-v_{i h}\right)-\sum_{e \in \mathcal{E}_{K}} \int_{e} \partial_{n_{K}} u_{i h}^{\varepsilon}\left(v_{i}-v_{i h}\right)\right) \\
& +\sum_{i=1}^{2}\left(f-f_{h}, v_{i}-v_{i h}\right)-\frac{1}{\varepsilon}\left[\left[u_{1 h}^{\varepsilon}-u_{2 h}^{\varepsilon}, v_{1}-v_{1 h}-\left(v_{2}-v_{2 h}\right)\right]\right]_{\Gamma} \cdot
\end{aligned}
$$

The first three summands in (25) are estimated in a standard way from the approximation properties (16) and (17) to give (see [16], Proposition 1.5)

$$
\begin{aligned}
\left(\left(\mathbf{u}^{\varepsilon}-\mathbf{u}_{h}^{\varepsilon}, \mathbf{v}\right)\right)_{\varepsilon} & \leq\left(\sum_{i=1}^{2} \sum_{K \in \mathcal{T}_{i h}}\left(\eta_{i}^{K}\right)^{2}+h_{K}^{2}|| f-\left.f_{h}\right|_{L^{2}(K)} ^{2}\right)^{1 / 2} \sum_{i=1}^{2}\left|v_{i}\right|_{1, \Omega_{i}} \\
& +\frac{1}{\varepsilon}\left|\left[\left[u_{1 h}^{\varepsilon}-u_{2 h}^{\varepsilon},\left(v_{1}-v_{1 h}\right)-\left(v_{2}-v_{2 h}\right)\right]\right]_{\Gamma}\right|
\end{aligned}
$$

To estimate the last term in (26), consider first the $L^{2}(\Gamma)$ penalty case. Using (17),

$$
\left|\left[\left[u_{1 h}^{\varepsilon}-u_{2 h}^{\varepsilon}, v_{1}-v_{1 h}-\left(v_{2}-v_{2 h}\right)\right]\right]_{\Gamma}\right| \leq\left\|u_{1 h}^{\varepsilon}-u_{2 h}^{\varepsilon}\right\|_{0, \Gamma} \sum_{i=1}^{2}\left\|v_{i}-v_{i h}\right\|_{0, \Gamma}
$$

whence

$$
\left|\left[\left[u_{1 h}^{\varepsilon}-u_{2 h}^{\varepsilon}, v_{1}-v_{1 h}-\left(v_{2}-v_{2 h}\right)\right]\right]_{\Gamma}\right| \leq C_{5} h^{1 / 2}|| u_{1 h}^{\varepsilon}-\left.u_{2 h}^{\varepsilon}\right|_{H_{00}^{1 / 2}(\Gamma)} \sum_{i=1}^{2}\left|v_{i}\right|_{1, \Omega_{i}}
$$

Consider now the $H_{00}^{1 / 2}(\Gamma)$ penalty case. Then,

$$
\left|\left[\left[u_{1 h}^{\varepsilon}-u_{2 h}^{\varepsilon}, v_{1}-v_{1 h}-\left(v_{2}-v_{2 h}\right)\right]\right]_{\Gamma}\right| \leq\left\|u_{1 h}^{\varepsilon}-u_{2 h}^{\varepsilon}\right\|_{H_{00}^{1 / 2}(\Gamma)} \sum_{i=1}^{2}\left\|v_{i}-v_{i h}\right\|_{H_{00}^{1 / 2}(\Gamma)},
$$

whence

$$
\left|\left[\left[u_{1 h}^{\varepsilon}-u_{2 h}^{\varepsilon}, v_{1}-v_{1 h}-\left(v_{2}-v_{2 h}\right)\right]\right]_{\Gamma}\right| \leq C_{6}|| u_{1 h}^{\varepsilon}-u_{2 h}^{\varepsilon} \|_{H_{00}^{1 / 2}(\Gamma)} \sum_{i=1}^{2}\left|v_{i}\right|_{1, \Omega_{i}}
$$


To conclude the proof, we need to use

$$
\sum_{i=1}^{2}\left|w_{i}\right|_{1, \Omega_{i}} \leq \sup _{\mathbf{v} \in \mathbf{X}} \frac{((\mathbf{w}, \mathbf{v}))_{\varepsilon}}{((\mathbf{v}, \mathbf{v}))_{\varepsilon}}, \quad \forall \mathbf{w}=\left(w_{1}, w_{2}\right) \in \mathbf{X}
$$

and next to combine (26) with either (27) or (28).

Q.E.D.

We next analyze the optimality of the error indicators that we have introduced. To do it, we need some further notation. For a given element $K \in \mathcal{T}_{i h}, i=1,2$, we define the union $\omega_{K}$ of the elements $K^{\prime} \in \mathcal{T}_{1 h} \cup \mathcal{T}_{2 h}$ that share an edge $(d=2)$ or a face $(d=3)$ with $K$. We denote by $\mathcal{E}_{K}^{\Gamma}$ (respectively, $\mathcal{E}_{K}^{0}$ ) the set of edges or faces of $\mathcal{E}_{K}$ that are (resp., are not) included in $\Gamma$. We also define the seminorm

$$
|\mathbf{v}|_{1, \omega_{K}, h}=\left(\sum_{i=1}^{2} \sum_{K \in \mathcal{T}_{i h}, K \subset \omega_{K}}\left|u_{i}^{\varepsilon}-u_{i h}^{\varepsilon}\right|_{1, K}^{2}\right)^{1 / 2} .
$$

We set the following result.

Theorem 6 The error indicators $\eta_{i}^{K}$ and $\eta_{h}^{P}$ verify the following estimates

$$
\begin{aligned}
\eta_{i}^{K} & \leq C\left(\left|\mathbf{u}^{\varepsilon}-\mathbf{u}_{h}^{\varepsilon}\right|_{1, \omega_{K}, h}+h_{K}|| f-\left.f_{h}\right|_{L^{2}\left(\omega_{K}\right)}\right), \\
\eta_{h}^{P} & \leq C^{\prime} \sum_{i=1}^{2}\left|u-u_{i h}^{\varepsilon}\right|_{1, \Omega_{i}},
\end{aligned}
$$

for some constants $C>0$ and $C^{\prime}>0$ that are independent on $\varepsilon$ and $h$.

\section{Proof.}

- Discretization error indicator. We start from the residual equation (25), where we set $v_{i h}=0$ :

$$
\begin{aligned}
\left(\left(\mathbf{u}^{\varepsilon}-\mathbf{u}_{h}^{\varepsilon}, \mathbf{v}\right)\right)_{\varepsilon} & =\sum_{i=1}^{2}\left(\sum_{K \in \mathcal{T}_{i h}} \int_{K}\left(f_{h}+\Delta u_{i h}^{\varepsilon}\right) v_{i}+\left(f-f_{h}, v_{i}\right)_{\Omega_{i}}\right) \\
& -\sum_{i=1}^{2} \sum_{K \in \mathcal{T}_{i h}}\left(\frac{1}{2} \sum_{e \in \mathcal{E}_{K}^{0}} \int_{e}\left[\partial_{n_{K}} u_{i h}^{\varepsilon}\right] v_{i}-\sum_{e \in \mathcal{E}_{K}^{\Gamma}} \int_{e} \partial_{n_{K}} u_{i h}^{\varepsilon} v_{i}\right) \\
& -\frac{1}{\varepsilon}\left[\left[u_{1 h}^{\varepsilon}-u_{2 h}^{\varepsilon}, v_{1}-v_{2}\right]\right]_{\Gamma} .
\end{aligned}
$$

For each $K \in \mathcal{T}_{i h}$, the estimate of $\left\|f_{h}+\Delta u_{i h}^{\varepsilon}\right\|_{L^{2}(K)}$ is standard. To do it, $v_{i}$ must be chosen as the product of $\left(f_{h}+\Delta u_{i h}^{\varepsilon}\right)_{\left.\right|_{K}}$ times the bubble function (the product of 
the barycentric coordinates of $K$ ) on element $K$ and to zero elsewhere. The use of inverse inequalities allows to prove that (Cf. Verfürth [16], Proposition 1.5)

$$
h_{K}\left\|f_{h}+\Delta u_{i h}^{\varepsilon}\right\|_{L^{2}(K)} \leq C_{1}\left(\left|\mathbf{u}^{\varepsilon}-\mathbf{u}_{h}^{\varepsilon}\right|_{H^{1}(K)}+h_{K}\left\|f-f_{h}\right\|_{L^{2}(K)}\right) .
$$

Next, let us estimate $\left\|\left[\partial_{n} u_{i h}^{\varepsilon}\right]\right\|_{L^{2}(e)}$, for some $e \in \mathcal{E}_{K}^{\Gamma}$. There exist two elements $K_{1} \in \mathcal{T}_{1 h}$ and $K_{2} \in \mathcal{T}_{2 h}$ that share the edge or face $e$. We choose $v_{i} \in X_{i}$ with support in $K_{i}$ such that $v_{\left.1\right|_{\Gamma}}=v_{\left.2\right|_{\Gamma}}=\varphi$, where $\varphi$ is a suitable polynomial function that we choose later. Then the interface product vanishes in the residual equation (31), that reduces to

$$
\left(\left(\mathbf{u}^{\varepsilon}-\mathbf{u}_{h}^{\varepsilon}, \mathbf{v}\right)\right)_{\varepsilon}=\sum_{i=1}^{2}\left(\int_{\partial K_{i}}\left[\partial_{n_{K}} u_{i h}^{\varepsilon}\right] v_{i}+\int_{K_{i}}\left(f_{h}+\Delta u_{i h}^{\varepsilon}\right) v_{i}+\left(f-f_{h}, v_{i}\right)\right) .
$$

The choice of the $v_{i}$ is standard: If $\psi_{e}$ denotes the bubble function on $e$, defined as the product of the barycentric coordinates of $e$, then

$$
\varphi=\left[\partial_{n_{K}} u_{i h}^{\varepsilon}\right] \psi_{e}
$$

This is a polynomial function of degree, say $r$, vanishing on $\partial e$. Then, the $v_{i}$ are defined by

$$
v_{i}=\left\{\begin{array}{lr}
\mathcal{R}_{K_{i}, e}(\varphi) & \text { in } K_{i} \\
0 & \text { in } \bar{\Omega} \backslash\left(K_{1} \cup K_{2}\right)
\end{array},\right.
$$

where $\mathcal{R}_{K_{i}, e}$ is a lifting operator from the space of polynomials $P_{r}(e)$ vanishing on $\partial e$ onto the space of polynomials $P_{r}\left(K_{i}\right)$ vanishing on $\partial K_{i} \backslash e$. This operator is constructed from a fixed lifting operator on a reference triangle by affine transformation. It verifies the estimate (Cf. Verfürth [16], Lemma 1.3 and Proposition 1.5)

$$
\left|\mathcal{R}_{K_{i}, e}(\varphi)\right|_{H^{1}(K)}+h_{K}^{-1}\left\|\mathcal{R}_{K_{i}, e}(\varphi)\right\|_{L^{2}(K)} \leq C_{2} h_{e}^{-1 / 2}\|\varphi\|_{L^{2}(e)} .
$$

From this estimate, again standard arguments allow to deduce from (33) that

$$
\begin{aligned}
h_{e}^{1 / 2}\left\|\left[\partial_{n} u_{i h}^{\varepsilon}\right]\right\|_{L^{2}(e)} & \leq C_{3}\left(\left|\mathbf{u}^{\varepsilon}-\mathbf{u}_{h}^{\varepsilon}\right|_{H^{1}\left(K_{1} \cup K_{2}\right)}+h_{K}\left\|f_{h}+\Delta u_{i h}^{\varepsilon}\right\|_{L^{2}\left(K_{1} \cup K_{2}\right)}\right. \\
& \left.+h_{K}\left\|f-f_{h}\right\|_{L^{2}\left(K_{1} \cup K_{2}\right)}\right) .
\end{aligned}
$$

The same choice of the $v_{i}$ allows to estimate $\left\|\left[\partial_{n} u_{i h}^{\varepsilon}\right]\right\|_{L^{2}(e)}$ for edges faces $e \in \mathcal{E}_{K}$ not situated on $\Gamma$. In this case the support of the $v_{i}$ does not intersect $\Gamma$, so the scalar product interface term in the expression of the residual directly vanishes.

Estimate (29) follows.

- Penalty error estimator. The estimate for $\eta_{h}^{P}$ is also obtained by a triangle inequality, as in Theorem 2,

$$
\eta_{h}^{P} \leq\left\|u_{1 h}^{\varepsilon}-\left.u\right|_{H_{00}^{1 / 2}(\Gamma)}+\right\| u_{2 h}^{\varepsilon}-u \|_{H_{00}^{1 / 2}(\Gamma)} \leq C_{4} \sum_{i=1}^{2}\left|u-u_{i h}^{\varepsilon}\right|_{1, \Omega_{i}} .
$$


Q.E.D.

Note that the estimator $\eta_{i}^{K}$ is not directly bounded in (29) in terms of the total error $\left|\mathbf{u}-\mathbf{u}_{h}^{\varepsilon}\right|_{1, \omega_{K}, h}$, but in terms of the discretization error $\left|\mathbf{u}^{\varepsilon}-\mathbf{u}_{h}^{\varepsilon}\right|_{1, \omega_{K}, h}$. This should not be surprising, as $\eta_{i}^{K}$ is the error arising in the discretization step of the penalized problem $\left(P_{\varepsilon}\right)$ to obtain $\left(P_{\varepsilon, h}\right)$.

Let us consider the penalty error $E_{\varepsilon}$ and the discretization error $E_{h}$, respectively defined by

$$
E_{\varepsilon}=\sum_{i=1}^{2}\left|u-u_{i}^{\varepsilon}\right|_{1, \Omega_{i}}, \quad E_{h}=\sum_{i=1}^{2}\left|u_{i}^{\varepsilon}-u_{i h}^{\varepsilon}\right|_{1, \Omega_{i}} .
$$

Theorems 5 and 6 set the following bounds (assuming for simplicity that there is no error stemming from the source terms),

$$
\begin{aligned}
C_{1}\left\{\eta_{h}^{P}+\left(\sum_{i=1}^{2} \sum_{K \in \mathcal{T}_{i h}}\left(\eta_{i}^{K}\right)^{2}\right)^{1 / 2}\right\} & \leq E_{\varepsilon}+E_{h} \\
& \leq C_{2}\left\{\eta_{h}^{P}+\left(\sum_{i=1}^{2} \sum_{K \in \mathcal{T}_{i h}}\left(\eta_{i}^{K}\right)^{2}\right)^{1 / 2}+\frac{h^{\gamma}}{\varepsilon} \eta_{h}^{P}\right\} .
\end{aligned}
$$

where $C_{1}$ and $C_{2}$ are constants independent of $h$ and $\varepsilon$. From these bounds we conclude that our error indicators are quasi-optimal. There is only a lack of optimality due to the $h^{\gamma} / \varepsilon$ term in the upper bound.

However, under some mild additional conditions we may considerably improve the estimate on the right-hand side of (34).

We introduce the following notation:

- $\pi_{i h}^{\Gamma}$ denotes the orthogonal projection operator from either $L^{2}(\Gamma)$ or $H_{00}^{1 / 2}(\Gamma)$ onto $W_{i h}$ with respect to the $[[\cdot, \cdot]]_{\Gamma}$ scalar product.

- For some $K \in \mathcal{T}_{i h}, \Delta_{K}$ denotes the set of all elements $K^{\prime}$ of $\mathcal{T}_{i h}$ such that $K \cap K^{\prime} \neq \emptyset$.

- For any face or side $e$ of some element $K \in \mathcal{T}_{i h}, \Delta_{e}$ denotes the set of all elements $K^{\prime}$ of $\mathcal{T}_{\text {ih }}$ such that $e \cap K^{\prime} \neq \emptyset$.

- $\mathcal{V}_{i}$ denotes the set of Lagrange interpolation nodes contained in $\bar{\Omega}_{i}$ associated with the space $X_{i h}$.

- $\mathcal{V}_{i}^{0}$ denotes the subset of the nodes of $\mathcal{V}_{i}$ located in $\Omega_{i}$.

- $\mathcal{V}_{i}^{\Gamma}$ denotes the subset of the nodes of $\mathcal{V}_{i}$ located on $\Gamma$.

- $\mathcal{T}_{i h}^{\Gamma}$ is the set of elements of $\mathcal{T}_{i h}$ that intersect $\Gamma$. 
- $\mathcal{T}_{i h}^{0}$ is the set of elements of $\mathcal{T}_{\text {ih }}$ that do not intersect $\Gamma$.

With each $a$ in $\mathcal{V}_{i}$, we associate the Lagrange function $\varphi_{a}$ in $X_{i h}$ which is equal to 1 in $a$ and to 0 in all $a^{\prime} \neq a$ in $\mathcal{V}_{i}$. Thus, we consider the Clément interpolation operators

$$
\mathcal{C}_{i h}^{*} v=\sum_{a \in \mathcal{V}_{i}}\left(\pi_{a} v\right)(a) \varphi_{a}
$$

where $\pi_{a}$ is a local $L^{2}$ projection operator (for instance, on an element $K_{a}$ of $\mathcal{T}_{i h}$ containing a). We also consider the modified Clément operator

$$
\mathcal{C}_{i h} v=\sum_{a \in \mathcal{V}_{i}^{0}}\left(\pi_{a} v\right)(a) \varphi_{a}+\sum_{a \in \mathcal{V}_{i}^{\Gamma}}\left(\pi_{i h}^{\Gamma} v\right)(a) \varphi_{a} .
$$

We state the following result.

Lemma 1 For any function $v_{i}$ of $H^{1}\left(\Omega_{i}\right)$, the following estimates hold

$$
\begin{gathered}
\left\|v_{i}-\mathcal{C}_{i h} v_{i}\right\|_{L^{2}(K)}^{2} \leq C h_{K}\left|v_{i}\right|_{H^{1}\left(\Delta_{K}\right)}, \quad \text { if } K \cap \Gamma=\emptyset ; \\
\left\|v_{i}-\mathcal{C}_{i h} v_{i}\right\|_{L^{2}(e)}^{2} \leq C h_{e}^{1 / 2}\left|v_{i}\right|_{H^{1}\left(\Delta_{e}\right)}, \quad \text { if } e \cap \Gamma=\emptyset ; \\
\left(\sum_{K \in \mathcal{T}_{i h}^{\Gamma}} h_{K}^{-2}\left\|v_{i}-\mathcal{C}_{i h} v_{i}\right\|_{L^{2}(K)}^{2}\right)^{\frac{1}{2}} \leq C\left(1+\lambda_{h}\right)\left|v_{i}\right|_{H^{1}\left(\Omega_{i}\right)} ; \\
\left(\sum_{K \in \mathcal{T}_{i h}^{\Gamma}} \sum_{e \in \mathcal{E}_{K}} h_{e}^{-1}\left\|v_{i}-\mathcal{C}_{i h} v_{i}\right\|_{L^{2}(e)}^{2}\right)^{\frac{1}{2}} \leq C\left(1+\lambda_{h}\right)\left|v_{i}\right|_{H^{1}\left(\Omega_{i}\right)} ;
\end{gathered}
$$

where

i) In the case of $L^{2}(\Gamma)$ projection,

$$
\lambda_{h}=\left(h_{M} / h_{m}\right)^{1 / 2}, \quad \text { with } \quad h_{M}=\max _{K \cap \Gamma \neq \emptyset} h_{K}, \quad h_{m}=\min _{K \cap \Gamma \neq \emptyset} h_{K} ;
$$

ii) In the case of $H_{00}^{1 / 2}(\Gamma)$ projection,

$$
\lambda_{h}=\left(h_{M} / h_{m}+h_{m}^{-1}\right)^{1 / 2} .
$$

We report the proof of this Lemma to an appendix to keep on the main line of our argumentation. Note that in the case of $L^{2}(\Gamma)$ projection, the coefficient $\lambda_{h}$ is bounded if only a finite number of grid refinements are performed, as it is usual. However, in the case of $H_{00}^{1 / 2}(\Gamma)$ projection, there is a $h_{m}^{-1 / 2}$ loss of optimality. Nevertheless, this loss is limited to the elements, sides or faces that intersect the interface $\Gamma$.

From the technical point of view, this loss of optimality could be avoided by means of a duality estimate of the error $\left\|v_{i}-\pi_{i h}^{\Gamma} v_{i}\right\|_{L^{2}(\Gamma)}$ in terms of the $H_{00}^{1 / 2}(\Gamma)$ norm of $v_{i}$, that is lacking up to our knowledge.

As a consequence of this Lemma, we deduce our main a posteriori error estimate result, 
Theorem 7 Assume that the trace spaces $W_{1 h}$ and $W_{2 h}$ defined in (19) coincide. Then, the solution of the discrete penalty problem $\left(P_{h, \varepsilon}\right)$ satisfies the following a posteriori error estimate

$$
\sum_{i=1}^{2}\left|u-u_{i h}^{\varepsilon}\right|_{1, \Omega_{i}} \leq C\left\{\eta_{h}^{P}+\left(\sum_{i=1}^{2} \sum_{K \in \mathcal{T}_{i h}}\left(\mu_{K} \eta_{i}^{K}\right)^{2}+\left(\mu_{K} h_{K}\right)^{2}\left\|f-f_{h}\right\|_{L^{2}(K)}^{2}\right)^{1 / 2}\right\}
$$

where $\mu_{K}=\left\{\begin{aligned}\left(1+\lambda_{h}\right) & \text { if } K \cap \Gamma \neq \emptyset, \\ 1 & \text { if } K \cap \Gamma=\emptyset .\end{aligned}\right.$

Proof. We adapt the proof of Theorem 5, that we only change from the residual equation (25) between problems $\left(P_{\varepsilon}\right)$ and $\left(P_{h, \varepsilon}\right)$.

Consider $v_{i} \in X_{i}, i=1,2$. We put $v_{i h}=\mathcal{C}_{i h} v_{i}$ in the residual equation (25). By construction of $\mathcal{C}_{i h}$, the trace on $\Gamma$ of $v_{i h}$ is $\pi_{i h}^{\Gamma} v_{i_{\Gamma}}$ that belongs to $W_{i h}$. As $W_{1 h}=W_{2 h}$, then $u_{1 h}^{\varepsilon}-u_{2 h}^{\varepsilon}$ belongs to $W_{i h}$, and it is orthogonal to $\left\{\left(v_{1}-v_{1 h}\right)-\left(v_{2}-v_{1 h}\right)\right\}_{\left.\right|_{\Gamma}}$ with respect to the $[[\cdot, \cdot]]_{\Gamma}$ scalar product. Then, the boundary term in the residual equation vanishes and this reduces to

$$
\begin{aligned}
\left(\left(\mathbf{u}^{\varepsilon}-\mathbf{u}_{h}^{\varepsilon}, \mathbf{v}\right)\right)_{\varepsilon} & =\sum_{i=1}^{2} \sum_{K \in \mathcal{T}_{i h}}\left(\int_{K}\left(f_{h}+\Delta u_{i h}^{\varepsilon}\right)\left(v_{i}-\mathcal{C}_{i h} v_{i}\right)-\sum_{e \in \mathcal{E}_{K}} \int_{e} \partial_{n_{K}} u_{i h}^{\varepsilon}\left(v_{i}-\mathcal{C}_{i h} v_{i}\right)\right) \\
& +\sum_{i=1}^{2}\left(f-f_{h}, v_{i}-\mathcal{C}_{i h} v_{i}\right)=I+I I+I I I
\end{aligned}
$$

We bound the first term of the right-hand side of (40) by

$$
\begin{aligned}
I & \leq C_{1} \sum_{i=1}^{2} \sum_{K \in \mathcal{T}_{i h}}\left\|f_{h}+\Delta u_{i h}^{\varepsilon}\right\|_{L^{2}(K)}^{2}\left\|v-\mathcal{C}_{i h} v_{i}\right\|_{L^{2}(K)} \\
& \leq C_{2}\left(\sum_{i=1}^{2} \sum_{K \in \mathcal{T}_{i h}^{\Gamma}}\left(\mu_{K} \eta_{K}\right)^{2}\right)^{1 / 2}\left(\sum_{i=1}^{2} \sum_{K \in \mathcal{T}_{i h}^{\Gamma}}\left(\mu_{K} h_{K}\right)^{-2}\left\|v_{i}-\mathcal{C}_{i h} v_{i}\right\|_{L^{2}(K)}^{2}\right)^{1 / 2} \\
& +C_{2}\left(\sum_{i=1}^{2} \sum_{K \in \mathcal{T}_{i h}^{0}} \eta_{K}^{2}\right)^{1 / 2}\left(\sum_{i=1}^{2} \sum_{K \in \mathcal{T}_{i h}^{0}} h_{K}^{-2}\left\|v_{i}-\mathcal{C}_{i h} v_{i}\right\|_{L^{2}(K)}^{2}\right)^{1 / 2} \\
& \leq C_{3}\left(\sum_{i=1}^{2} \sum_{K \in \mathcal{T}_{i h}}\left(\mu_{K} \eta_{K}\right)^{2}\right)^{1 / 2}\left(\sum_{i=1}^{2}\left|v_{i}\right|^{2}\right.
\end{aligned}
$$

where the last bound comes from estimates (35) and (37) of Lemma 1. Similar arguments lead to the bound for the quantity $I I I$. The second term of the right-hand side of (40) is 
bounded by

$$
\begin{aligned}
I I & \leq \frac{1}{2} \sum_{i=1}^{2} \sum_{K \in \mathcal{T}_{i h}} \sum_{e \in \mathcal{E}_{K}}\left\|\left[\partial_{n_{K}} u_{i h}^{\varepsilon}\right]\right\|_{L^{2}(e)}\left\|v_{i}-\mathcal{C}_{i h} v_{i}\right\|_{L^{2}(e)} \\
& \leq C_{4}\left(\sum_{i=1}^{2} \sum_{K \in \mathcal{T}_{i h}^{\Gamma}}\left(\mu_{K} \eta_{K}\right)^{2}\right)^{1 / 2}\left(\sum_{i=1}^{2} \sum_{K \in \mathcal{T}_{i h}^{\Gamma}} \sum_{e \in \mathcal{E}_{K}} \mu_{K}^{-2} h_{e}^{-1}\left\|v_{i}-\mathcal{C}_{i h} v_{i}\right\|_{L^{2}(e)}^{2}\right)^{1 / 2} \\
& +C_{4}\left(\sum_{i=1}^{2} \sum_{K \in \mathcal{T}_{i h}^{0}} \eta_{K}^{2}\right)^{1 / 2}\left(\sum_{i=1}^{2} \sum_{K \in \mathcal{T}_{i h}^{0}} \sum_{e \in \mathcal{E}_{K}} h_{e}^{-1}\left\|v_{i}-\mathcal{C}_{i h} v_{i}\right\|_{L^{2}(e)}^{2}\right)^{1 / 2} \\
& \leq C_{5}\left(\sum_{i=1}^{2} \sum_{K \in \mathcal{T}_{i h}}\left(\mu_{K} \eta_{K}\right)^{2}\right)^{1 / 2}\left(\sum_{i=1}^{2}\left|v_{i}\right|^{2}\right)^{1 / 2}
\end{aligned}
$$

where the last bound comes from estimates (36) and (38) of Lemma 1.

The remaining of the proof is similar to that of Theorem 5 . Q.E.D. 


\section{Improvement of convergence rate}

If $H_{00}^{1 / 2}(\Gamma)$ penalty is used, the convergence rate of the parallel iteration process (14) is remarkably increased. This is proved thanks to Theorem 4 by replacing the $L^{2}(\Gamma)$ penalty by the $H_{00}^{1 / 2}(\Gamma)$ one in the proof of Theorem 2 in [7]. This yields

$$
\left\|u_{1 h}^{\varepsilon}-u_{2 h}^{\varepsilon}\right\|_{H_{00}^{1 / 2}(\Gamma)} \leq C\left(\sqrt{\varepsilon} h^{k}+\varepsilon\right),
$$

where $C=C(u)$ is a positive constant that only depends on $u$. Combining this estimate with (20), we deduce

$$
\sum_{i=1}^{2}\left|u_{h}^{\varepsilon}-u_{i h}^{\varepsilon}\right|_{1, \Omega_{i}} \leq C\left(\sqrt{\varepsilon} h^{k}+\varepsilon\right)
$$

Note that

$$
\sum_{i=1}^{2}\left|u_{i}-u_{i h}^{\varepsilon}\right|_{1, \Omega_{i}} \leq \sum_{i=1}^{2}\left|u_{i}-u_{i}^{\varepsilon}\right|_{1, \Omega_{i}}+\sum_{i=1}^{2}\left|u_{i}^{\varepsilon}-u_{h}^{\varepsilon}\right|_{1, \Omega_{i}}+\sum_{i=1}^{2}\left|u_{h}^{\varepsilon}-u_{i h}^{\varepsilon}\right|_{1, \Omega_{i}},
$$

where the second term in the right-hand side is of order $h^{k}$. Then, the best choice for $\varepsilon$ in (41)

$\varepsilon=\mathrm{O}\left(h^{k}\right)$, to achieve an overall $\mathrm{O}\left(h^{k}\right)$ accuracy. Other choices improving the accuracy between $u_{h}^{\varepsilon}$ and $u_{i h}^{\varepsilon}$ would require a larger computational effort without diminishing the discretization error which in any case is of order $h^{k}$. This choice $\varepsilon=\mathrm{O}\left(h^{k}\right)$ gives

$$
\sum_{i=1}^{2}\left|u_{h}-u_{i h}^{\varepsilon}\right|_{1, \Omega_{i}} \leq C h^{k}, \quad \text { and then } \quad \sum_{i=1}^{2}\left|u-u_{i h}^{\varepsilon}\right|_{1, \Omega_{i}} \leq C h^{k} .
$$

This allows to state the

Theorem 8 Assume that hypothesis (15) on the solution u of problem (1) hold. Assume that the sequence $\left\{\left(u_{1 h}^{n+1, \varepsilon}, u_{2 h}^{n+1, \varepsilon}\right)\right\}_{n \geq 1}$ is computed by the full discretized problem $\left(P_{\varepsilon, h}^{n}\right)$, with $H_{00}^{1 / 2}(\Gamma)$ penalty and a penalty parameter $\varepsilon$ of size $\varepsilon=\mathcal{O}\left(h^{k}\right)$. Then, if the number of iterations $n$ is of order $\mathcal{O}\left(|\log h| h^{-k}\right)$, the following error estimate holds,

$$
\sum_{i=1}^{2}\left|u-u_{i h}^{n, \varepsilon}\right|_{1, \Omega_{i}} \leq C h^{k}
$$

where $C$ is a constant independent of $\varepsilon$ and $h$.

Proof. An argument similar to that developed in the proof of Theorem 3 of [7] yields:

$$
\sum_{i=1}^{2}\left|u_{i h}^{\varepsilon}-u_{i h}^{\varepsilon, n+1}\right|_{1, \Omega_{i}} \leq \frac{E_{0}}{\sqrt{\varepsilon}} \frac{1}{\left(1+2 C_{0} \varepsilon\right)^{n / 2}},
$$


where $E_{0}=\sum_{i=1}^{2}\left\|u_{i h}^{0, \varepsilon}-u_{i h}^{\varepsilon}\right\|_{0, \Gamma}$ and $C_{0}$ is a positive constant independent of $h$ and $\varepsilon$.

As $\varepsilon=\mathcal{O}\left(h^{k}\right)$, and the number of iterations $n$ is of order $\mathcal{O}\left(|\log h| h^{-k}\right)$ estimate (44) yields an accuracy of order $h^{k}$ between $u_{i h}^{\varepsilon}$ and $u_{i h}^{\varepsilon, n+1}$ in $H^{1}\left(\Omega_{i}\right)$ norm. It is enough to combine it with estimate (42) to obtain (43).

Q.E.D.

Remark 2 The number of iterations $\mathcal{O}\left(|\log h| h^{-k}\right)$ given by this result to obtain an $\mathcal{O}\left(h^{k}\right)$ accuracy with $H_{00}^{1 / 2}(\Gamma)$ penalty must be compared with a number of iterations of order $\mathcal{O}\left(|\log h| h^{-2 k}\right)$, which corresponds to $L^{2}(\Gamma)$ penalty. 


\section{Application to penalty parameter optimization and grid adaptation}

We describe a strategy to optimize the penalty parameter and to adapt the grid, based upon the error indicators developed in the previous sections. Our purpose is to determine the penalty parameter that yields an error of the same size or sligthly smaller than the error due to the discretization, for a fixed grid. Indeed, computing a numerical solution with a penalty parameter that yields a penalty error neatly smaller than that of the discretization would require a useless extra computational effort.

\section{Adaptation algorithm}

We use the penalty error and discretization error indicators (for both $H_{00}^{1 / 2}(\Gamma)$ and $L^{2}(\Gamma)$ penalties) by

$$
\begin{aligned}
\eta_{h}^{n+1, P} & =\left\|u_{1 h}^{n+1, \varepsilon}-u_{2 h}^{n+1, \varepsilon}\right\|_{H_{00}^{1 / 2}(\Gamma)}, \\
\eta_{h}^{n+1, D} & =\left(\sum_{i=1}^{2} \sum_{K \in \mathcal{T}_{i h}}\left(\eta_{i}^{n+1, K}\right)^{2}\right)^{1 / 2}, \text { with } \\
\eta_{i}^{n+1, K}=h_{K} \| f_{h} & +\Delta u_{i h}^{n+1, \varepsilon}\left\|_{L^{2}(K)}+\sum_{e \in \mathcal{E}_{K}} h_{e}^{1 / 2}\right\|\left[\partial_{n} u_{i h}^{n+1, \varepsilon}\right] \|_{L^{2}(e)} .
\end{aligned}
$$

Initialization: Set three tolerance levels $\eta_{1}, \eta_{2}$ and $\eta_{3}$ and a parameter $\rho$ in $[0,1]$.

- Set an initial penalty parameter $\varepsilon_{e}^{0}>0$.

- Build an initial triangulation $\mathcal{T}_{h}^{0}$ such that the corresponding approximation error $h\left\|f-f_{h}\right\|_{L^{2}(\Omega)}$ is smaller than $\eta_{1}$.

Iteration: Once solved the full discrete problem $\left(P_{\varepsilon, h}^{n}\right)$ with a current penalty error $\varepsilon^{n}$, compute the error indicators by (45) and (46) Then, the adaptation strategy is made in three steps:

* Step 1: Adaptation of the penalty parameter.

- If $\eta_{h}^{n+1, P}>\eta_{h}^{n+1, D}$, set $\varepsilon^{n+1}=\theta^{n} \varepsilon^{n}, \quad$ with $\theta^{n}=\frac{(1-\rho) \eta_{h}^{n+1, D}}{\eta_{h}^{n+1, P}}$;

- If $\eta_{h}^{n+1, P}<\eta_{h}^{n+1, D}$, set $\varepsilon^{n+1}=\varepsilon^{n}$.

* Step 2: Adaptation of the mesh.

We compute the $\eta_{i}^{K}$ and their mean value $\bar{\eta}_{h}$. For all $K$ such that $\eta_{i}^{K}$ is larger than $\bar{\eta}_{h}$, we divide $K$ into smaller triangles such that the diameters of these new elements behave like $h_{i}^{K}$ multiplied by the ratio $\bar{\eta}_{h} / \eta_{i}^{K}$. 
* Step 3: Solution of $\left(P_{\varepsilon, h}^{n}\right)$.

Iterate the Schwarz procedure to obtain a refined solution of $\left(P_{\varepsilon, h}^{n}\right)$, until

$$
\left\|\mathbf{u}_{h}^{m+1, \epsilon}-\mathbf{u}_{h}^{m, \epsilon}\right\|_{\mathbf{X}} /\left\|\mathbf{u}_{h}^{m, \epsilon}\right\|_{\mathbf{X}}<\eta_{2}, \quad \text { where } \quad \mathbf{u}_{h}^{m, \epsilon}=\left(u_{1 h}^{m, \epsilon}, u_{2 h}^{m, \epsilon}\right)
$$

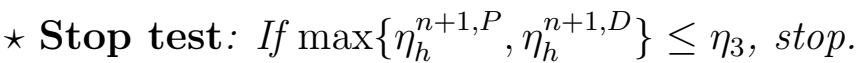

We refer to [3], Section 5, for first tests of this strategy in a complete different framework. In [3], Step 2 is iterated 4 of 5 times for each iteration of Step 1. In our case we have observed a faster convergence when Step 2 is iterated just once. Also, Step 3 in practice is needed to guarantee the convergence of the overall process, particularly for large grid sizes.

Optimization of penalty parameter:

By skipping Step 2, we only optimize the value of $\varepsilon$ for a fixed mesh. 


\section{$9 \quad$ Numerical experiments}

This section is aimed to two objectives: Comparing the practical efficiency of $H_{00}^{1 / 2}(\Gamma)$ versus $L^{2}(\Gamma)$ penalty, and testing the grid adaptation + penalty parameter optimization strategy. In a first step, we test the theoretical predictions of convergence rate and error estimates for the single Schwarz iteration process (14). Next we check the efficiency of the two types of indicators. In a third step, we propose an adaptive strategy aimed to optimize the choice of $\varepsilon$ with and without refining the mesh.

Our results are rather surprising: For a fixed grid the $H_{00}^{1 / 2}(\Gamma)$ penalty is faster than the $L^{2}(\Gamma)$ penalty when the penalty parameter $\varepsilon$ is small enough, as expected from the theoretical predictions. However, when a combined strategy of grid adaptation + penalty parameter optimization is used, the situation turns to be the opposite: The $L^{2}(\Gamma)$ penalty is faster, and the gain of computing time increases as $h$ decreases. This is mainly due to the cost required by the computation of the $H_{00}^{1 / 2}(\Gamma)$ norm. In fact, we use a discrete equivalent norm much less costly, but this does not changes our conclusions: The $H_{00}^{1 / 2}(\Gamma)$ penalty is faster for a fixed grid, but the $L^{2}(\Gamma)$ penalty is much faster when grid adaptation strategies are used.

In this section, we work in dimension $d=2$ and in the case where the discrete spaces are made of piecewise affine functions $\left(k_{1}=k_{2}=1\right)$. All experiments are performed on the finite element code FreeFEM++, see [10], using a Toshiba laptop computer with an Intel Pentium processor to $1.80 \mathrm{Ghz}$.

We recall that the scalar product of $H_{00}^{1 / 2}(\Gamma)$ as defined in (2) is non local, hence difficult to compute. So, in the numerical experiments that follow, we use instead a discrete analogue: If $e_{j}, 1 \leq j \leq J(h)$, denote the edges of $\mathcal{E}_{h}^{\Gamma}$ such that the endpoints of each $e_{j}$ are $x_{j}$ and $x_{j+1}$, and if $h_{j}$ stands for the length $\left|x_{j+1}-x_{j}\right|$,

$$
[[w, v]]_{h \Gamma}=\sum_{j=1}^{J(h)} h_{j} w^{j} v^{j}+\sum_{j=1}^{J(h)} \sum_{k=1, k \neq j}^{J(h)} h_{j} h_{k} \frac{\left(w^{j}-w^{k}\right)\left(v^{j}-v^{k}\right)}{\left(x_{j}-x_{k}\right)^{2}}+\sum_{j=1}^{J(h)} h_{j} \frac{w^{j} v^{j}}{d\left(x_{j}, \partial \Gamma\right)},
$$

where $w^{j}$ and $v^{j}$ denotes the values of $w$ and $v$ at the node $x_{j}$. Indeed, it is proved in [5], Lemma 5.1, that the norm associated with the two first terms in this summand is equivalent to the norm of $H^{1 / 2}(\Gamma)$ on the spaces $W_{1 h}$ and $W_{2 h}$, with equivalence constants independent of $h$, in the case of a uniformly regular family of triangulations. However the extension of this result to a regular family of triangulations and also to the $H_{00}^{1 / 2}(\Gamma)$ norm seems easy to establish.

We consider two test cases:

L-shaped domain. Here, $\Omega=] 0,1\left[2 \backslash\left[\frac{1}{2}, 1\right]^{2}\right.$. It is divided into two sub-domains symmetric with respect to the straight line $x=y$. The boundary $\Gamma$ is the segment of this line between the points $(0,0)$ and $(1,1)$. We consider the solution $u$ given by

$$
u(x, y)=\sin (3 x+y) x y(1-x)(1-y)\left(\frac{1}{2}-x\right)\left(\frac{1}{2}-y\right),
$$


which satisfies the homogeneous Dirichlet boundary condition, and is not symmetric with respect to the boundary $\Gamma$. Figure 3 displays one of the initial triangulations that we have used for this domain and its division into two sub-domains.

Square domain. Here, $\Omega$ is the square $] 0,1\left[2\right.$, divided into the rectangles $\Omega_{1}=$ ] $0,1[\times] 0, \frac{1}{2}\left[\right.$ and $\left.\Omega_{2}=\right] 0,1[\times] \frac{1}{2}, 1[$. We consider the solution $u$ given by

$$
u(x, y)=\sin (x y) x(1-x) y(1-y),
$$

which satisfies the desired boundary conditions.

We mainly present the results for the L-shape domain test, but we stress that all results that we report are qualitatively similar for the square domain test.

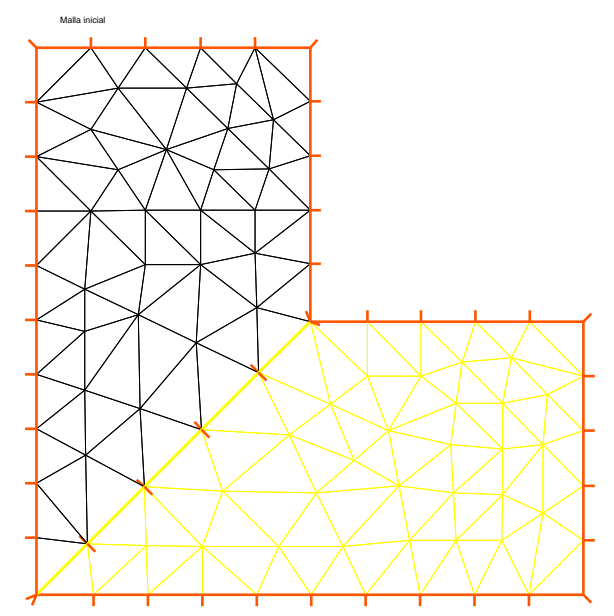

Figure 3: Initial triangulation of L-shaped domain.

\subsection{Test of a priori error analysis}

We test here the analysis of penalty error and the improvement of convergence rate performed in Sections 3 and 7, respectively.

Theorem 1 and Corollary 3 respectively state penalty error estimates proportional to $\sqrt{\epsilon}$ for $L^{2}(\Gamma)$ penalty and to $\epsilon$ for $H_{00}^{1 / 2}(\Gamma)$ penalty. We have observed that in practice these estimates are sharp, in the sense that the rate of $H_{00}^{1 / 2}(\Gamma)$ to $L^{2}(\Gamma)$ errors is proportional to $\sqrt{\epsilon}$. This probably corresponds to asymptotic behaviors as

$$
E_{H^{1 / 2}}(\epsilon) \simeq C \epsilon, \quad E_{L^{2}}(\epsilon) \simeq C^{\prime} \sqrt{\epsilon} \quad \text { as } \quad \epsilon \rightarrow 0
$$

Here we denote

$$
E_{H^{1 / 2}}(\epsilon)=\left\|\mathbf{u}-\mathbf{u}^{\epsilon}\right\|_{\mathbf{X}}, \quad E_{L^{2}}(\epsilon)=\left\|\mathbf{w}-\mathbf{w}^{\epsilon}\right\|_{\mathbf{X}},
$$

where $\mathbf{u}^{\epsilon}$ and $\mathbf{w}^{\epsilon}$ respectively are the solutions of $\left(P_{\epsilon}\right)$ for $H_{00}^{1 / 2}(\Gamma)$ and $L^{2}(\Gamma)$ penalty. 


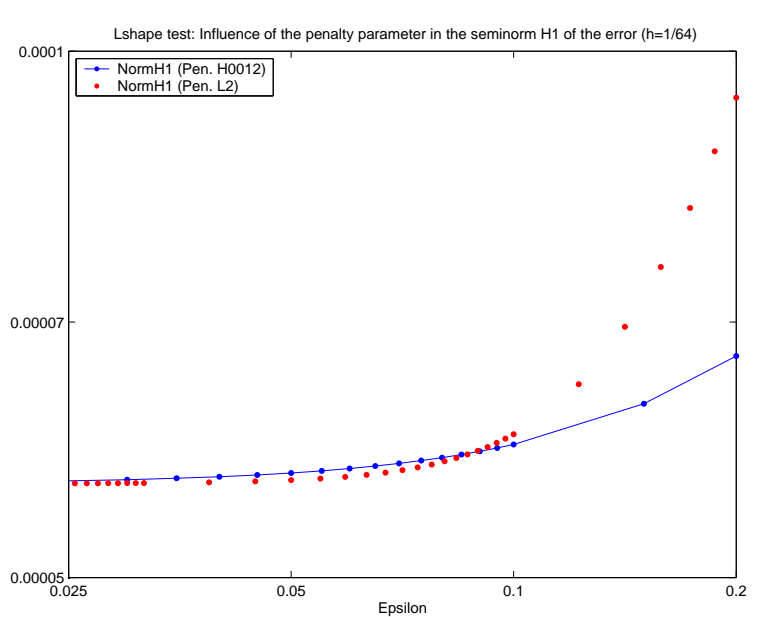

(a) L-shaped domain

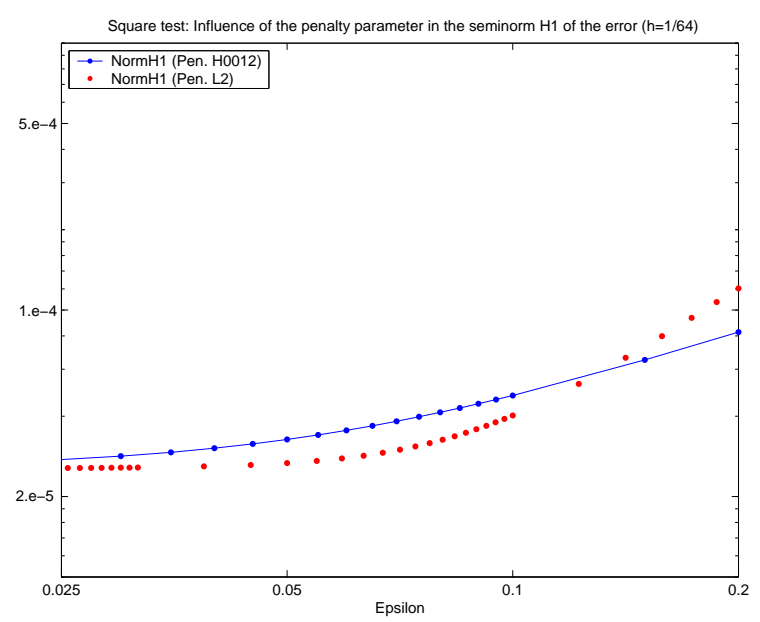

(b) Square domain

Figure 4: Comparison of penalty errors due to $H_{00}^{1 / 2}(\Gamma)$ penalty (for penalty parameter $\epsilon$ ) and to $L^{2}(\Gamma)$ (for penalty parameter $\epsilon^{2}$ ).

Figure 4 displays approximations of the computed errors $E_{H^{1 / 2}}(\epsilon)$ and $E_{L^{2}}\left(\epsilon^{2}\right)$ for the Lshape and square domains with grids of size $h=1 / 64$, as a function of $\epsilon$. We approximate $\mathbf{u}$ by its Lagrange interpolate $\mathcal{I}_{h} \mathbf{u}$ on space $V_{h}$. We also approximate for instance $\mathbf{u}^{\epsilon}$ by a close-to-convergence $\mathbf{u}_{h}^{m+1, \epsilon}$ in the iterative Schwarz process (14). Specifically, we have taken $\mathbf{u}^{\epsilon}=\mathbf{u}_{h}^{m+1, \epsilon}$ when the quantity

$$
\left\|\mathbf{u}_{h}^{m+1, \epsilon}-\mathbf{u}_{h}^{m, \epsilon}\right\|_{\mathbf{X}} /\left\|\mathbf{u}_{h}^{m, \epsilon}\right\|_{\mathbf{X}}<\eta=10^{-7}
$$

These errors indeed appear to be proportional (in fact, are almost equal) for $\epsilon$ small enough, in agreement with relations (49). We have observed the same qualitative behavior for several grids of both test domains. We just present one of these results for brevity. It is striking that these errors appear to be asymptotically equal instead of simply proportional, but we have obtained the same behavior in all our tests. We infer that the constants $C$ and $C^{\prime}$ in (49) are equal for some reason.

Also, as stated in Remark 2, the $H_{00}^{1 / 2}(\Gamma)$ penalty provides a better convergence rate than the $L^{2}(\Gamma)$ penalty in the Schwarz iterative process (14). To test this point, we have run both penalty procedures on a same grid, and compared the number of iterations needed for the iterative processes to converge.

Figure 5 displays the number of iterations required by the $H_{00}^{1 / 2}(\Gamma)$ and $L^{2}(\Gamma)$ penalty procedures for the L-shape and square domains test, as a function of $\epsilon$. Here again, we approximate $\mathbf{u}^{\epsilon}$ by the $\mathbf{u}_{\epsilon}^{m+1}$ verifying the stop test (50). We observe a linear dependence of this number of iterations with respect to $\epsilon$ for $L^{2}(\Gamma)$ and $H_{00}^{1 / 2}(\Gamma)$ penalty, with a better slope for the $H_{00}^{1 / 2}(\Gamma)$ one. We have observed exactly the same qualitative behavior for 


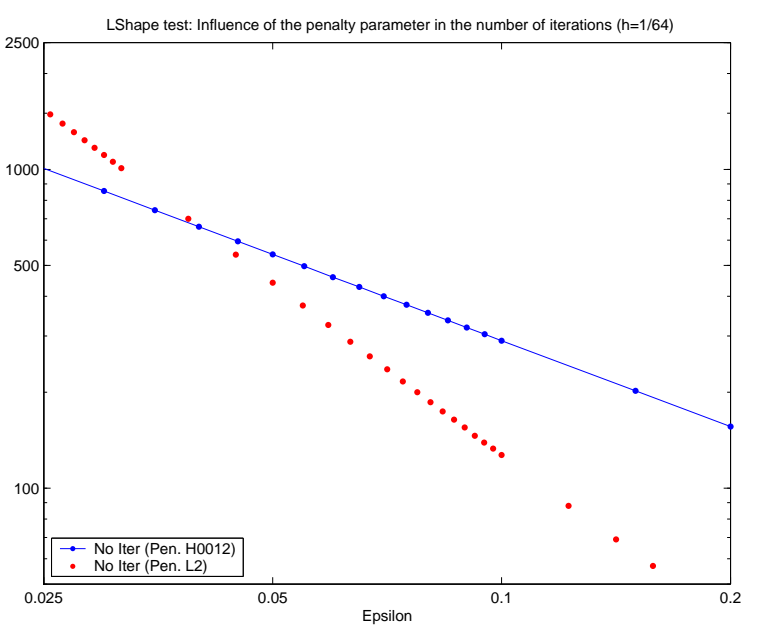

(a) L-shaped domain

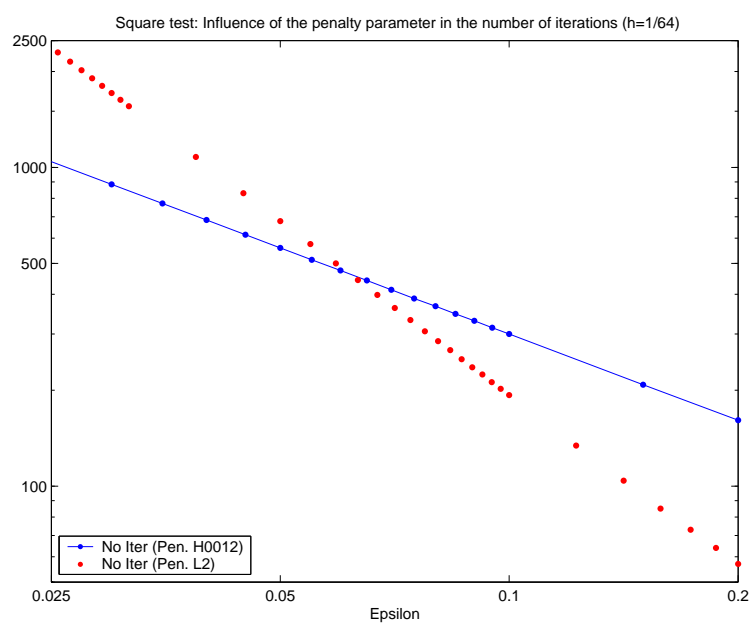

(b) Square domain

Figure 5: Comparison of number of iterations required for convergence of Schwarz iterative processes.

other grid sizes $h$. We conclude that for $\epsilon$ small enough the $H_{00}^{1 / 2}(\Gamma)$ penalty procedure is faster than the $L^{2}(\Gamma)$ one.

\subsection{The efficiency of the indicators}

To test the efficiency of the indicators, we first work with a uniform structured mesh for the square domain, with $h$ equal to $1 / 32$ and $\varepsilon$ decreasing from 5 to $5 \times 10^{-3}$. Figure 6 (a) presents, in logarithmic scales and as a function of $\varepsilon$,

- the curves of the errors

$$
\sum_{i=1}^{2}\left|\mathcal{I}_{h} \mathbf{u}-\mathbf{u}_{i h}^{\varepsilon}\right|_{H^{1}\left(\Omega_{i}\right)} \quad \text { and } \quad \sum_{i=1}^{2}\left\|\mathcal{I}_{h} \mathbf{u}-\mathbf{u}_{i h}^{\varepsilon}\right\|_{L^{2}\left(\Omega_{i}\right)},
$$

in dotted dashed line and dotted line, respectively.

- the indicator $\eta_{h}^{P}$ in plain line and the Hilbertian sum $\eta_{h}^{D}$ of all indicators $\eta_{i}^{K}$ in dashed line.

The error decreases with $\varepsilon$ until the penalization error (which behaves like $c \varepsilon$ ) is of the same order as the finite element discretization error, for a critical value $\varepsilon_{c} \simeq 0.2$. For $\varepsilon$ sufficiently larger than $\varepsilon_{c}$, the error indicator curve $\eta_{h}^{P}$ is parallel to the curves of the errors. In contrast, the quantity $\eta_{h}^{D}$ turns out to be fully independent of $\varepsilon$.

Next, we fix $\varepsilon$ equal to 0.01 and we still use uniform (unstructured) meshes. Figure 6(b) presents the same curves as previously, now in semi-logarithmic scales and for $h$ decreasing from 0.25 to $1.5 \times 10^{-2}$. There also the error decreases with $h$ until it reaches 


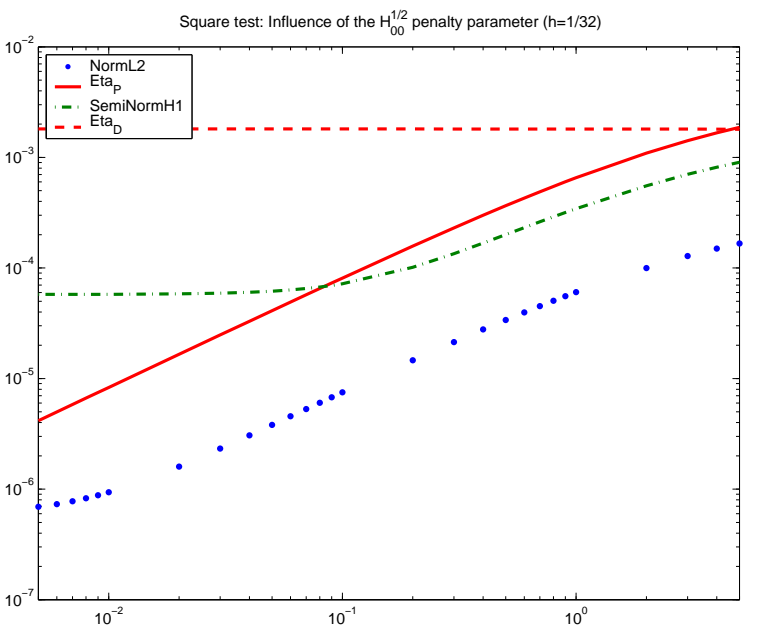

(a) Fixed grid

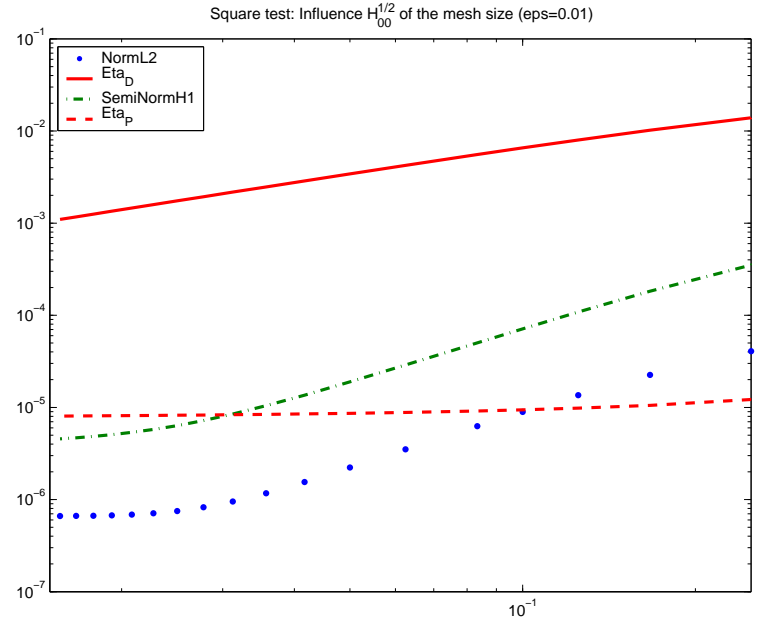

(b) Fixed epsilon

Figure 6: Influence of penalty parameter and grid size in error indicators: Square domain

the penalization error. Also, the curves of the indicators and of the errors are almost parallel for $h$ sufficiently larger than a critical $h_{c}$.

Figure 7 represents the same curves for the L-squared domain, where the behavior of the indicators is qualitatively the same. Let us also remark that the behavior of indicators corresponding to $L^{2}$ penalty is also similar, for both the square and the L-shaped domain.

We can infer from these results that the error estimate (39) is probably suboptimal, in the sense that the parameter $\mu_{K}$ appears in practice to be of order one. If this parameter had an asymptotic growth to $+\infty$ as $h \rightarrow 0$, we would not have obtained the behavior of the indicators exhibited in Figures $6(\mathrm{~b})$ and $7(\mathrm{~b})$.

These results resemble in a striking way to those obtained in [3], Section 5, for a completely different type of penalization. In this last paper, the Stokes problem is solved by non-mixed finite elements, and penalty is used to treat the incompressibility restriction. This similar behavior is probably due to the common formulation of both discretizations as mixed problems, in the way indicated in Section 4.

\subsection{Testing the adaptivity strategy and penalty parameter op- timization}

The adaptivity strategy provides a large saving of computational effort for both $H_{00}^{1 / 2}(\Gamma)$ and $L^{2}(\Gamma)$ penalties. The most surprising result is that the $L^{2}(\Gamma)$ penalty is computationally faster than the $H_{00}^{1 / 2}(\Gamma)$ one.

We present the results obtained with the L-shape domain test. Figures 8(a), 8(b) and 9 respectively display an intermediate solution, the final solution and the grids associated to these solutions. These results are obtained starting from the initial triangulation pre- 


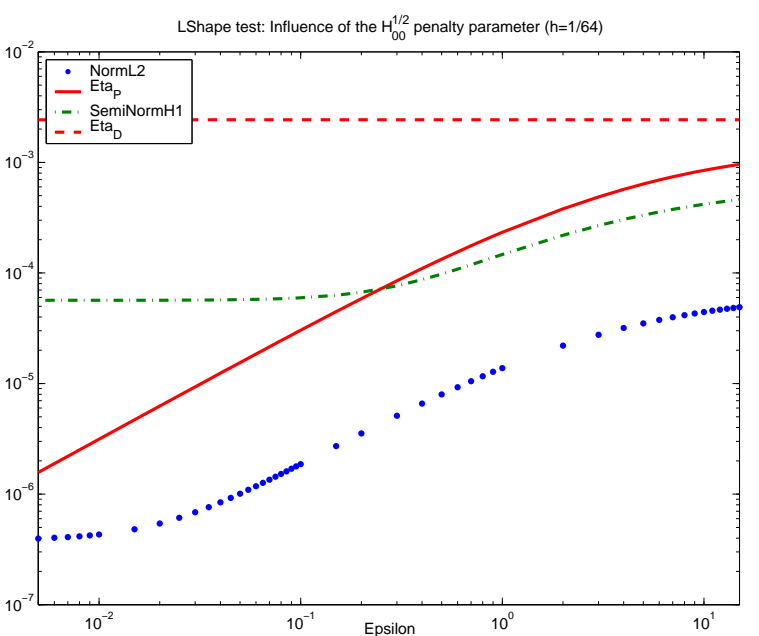

(a) Fixed grid

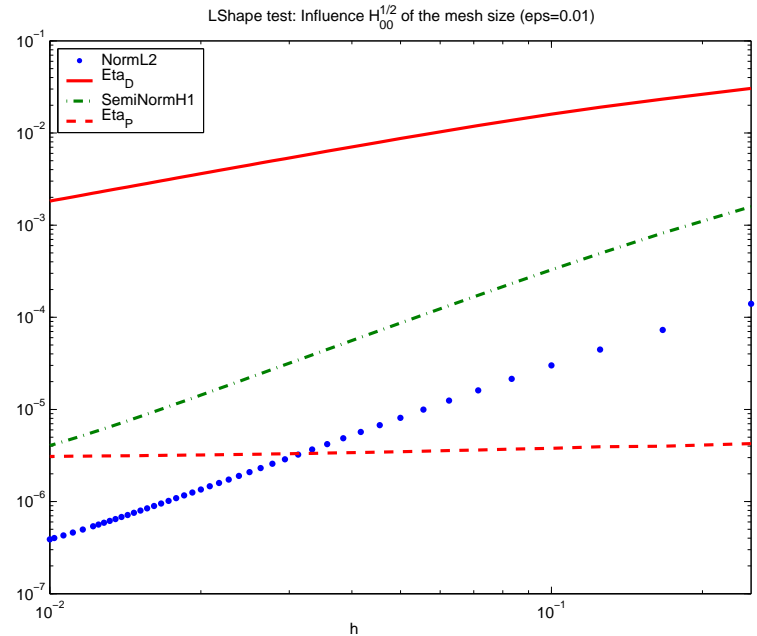

(b) Fixed epsilon

Figure 7: Influence of penalty parameter and grid size in error indicators: L-shaped domain

sented in Figure 3, with $H_{00}^{1 / 2}(\Gamma)$ penalty. We observe that the continuity and even the differentiability of iso-lines of the solution across the interface $\Gamma$ is progressively improved in the adaptive process. The intermediate solution could be acceptable for some applications not requiring high precision. The high accuracy of the final solution is rather costly, as the density of triangles of the adapted grid is quite large. This density is larger in the zones of strong gradients of the solution, as could be expected, but is not particularly high in the neighborhood of $\Gamma$.

\section{Combined grid adaptation-penalty parameter optimization}

In order to compare the $H_{00}^{1 / 2}(\Gamma)$ and $L^{2}(\Gamma)$ penalties, we have run the L-shape domain test with the same set of data for both: The initial grid displayed in Figure $3, \varepsilon^{0}=0.1$, $\eta_{1}=0.1, \eta_{2}=5 \times 10^{-2}, \rho=0.05$. To obtain an accurate solution, we have stopped the process when

$$
\left\|\mathbf{u}_{h}^{n, \epsilon}-\mathcal{I}_{h} \mathbf{u}\right\|_{\mathbf{X}} /\left\|\mathcal{I}_{h} \mathbf{u}\right\|_{\mathbf{X}}<3 \times 10^{-3} .
$$

Table 1 compares the results obtained. The number of iterations stands for the total number of applications of the Schwarz iteration procedure along the adaptation process. We observe that the values of the estimators $\eta_{h}^{P}$ and $\eta_{h}^{D}$ and the number of triangles of the final grids are quite close for both penalties. Also, that the optimal $\varepsilon$ for $L^{2}(\Gamma)$ penalty is close to the square of the optimal $\varepsilon$ for $H_{00}^{1 / 2}(\Gamma)$ penalty. We may observe a large saving of CPU time for $L^{2}(\Gamma)$ penalty. This is due to two effects: The faster convergence of the $L^{2}(\Gamma)$ penalty, that requires much less iterations, and the cost due to the computation of the (even discrete) $H_{00}^{1 / 2}(\Gamma)$ norm. 


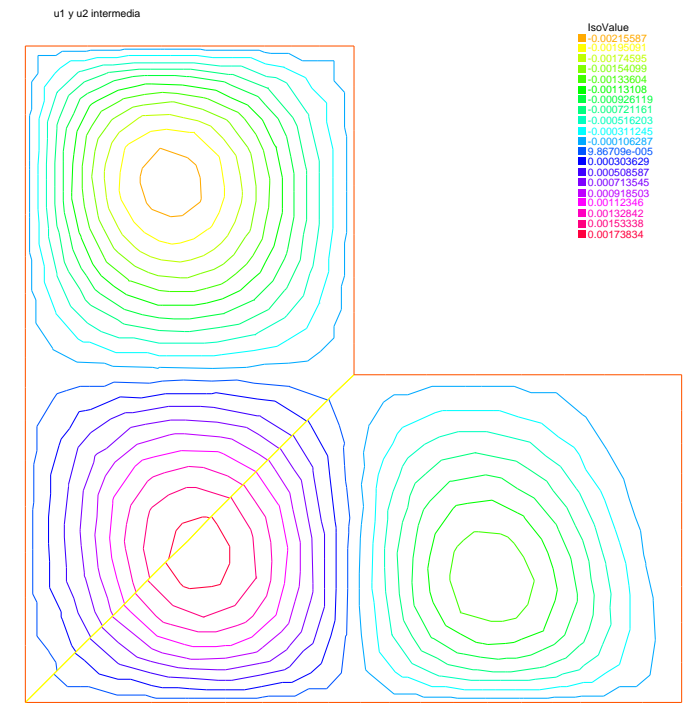

(a) Intermediate solution

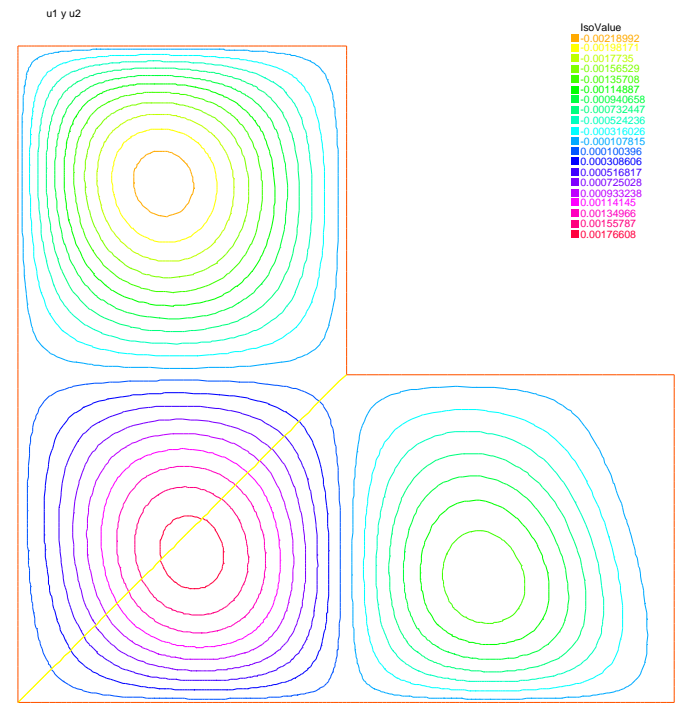

(b) Final solution

Figure 8: Snapshots of iterated solutions in adaptivity strategy

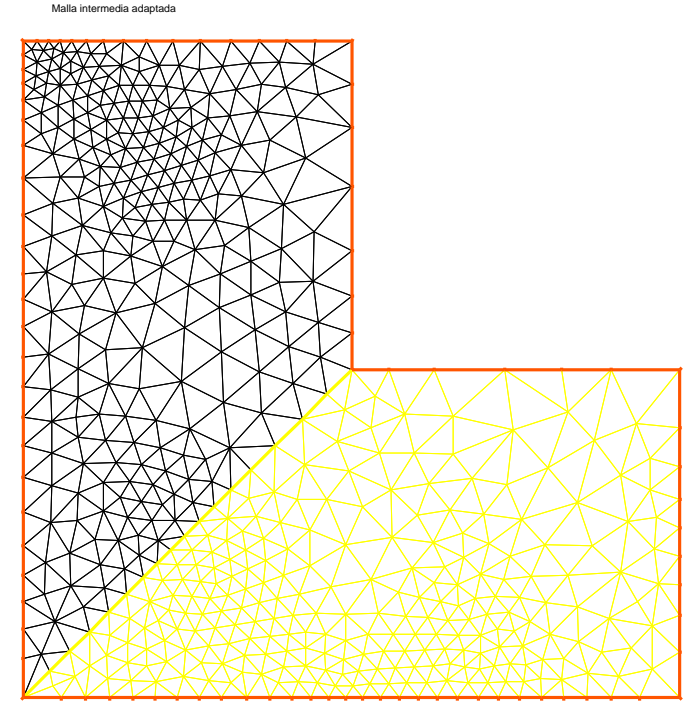

(a) Intermediate grid

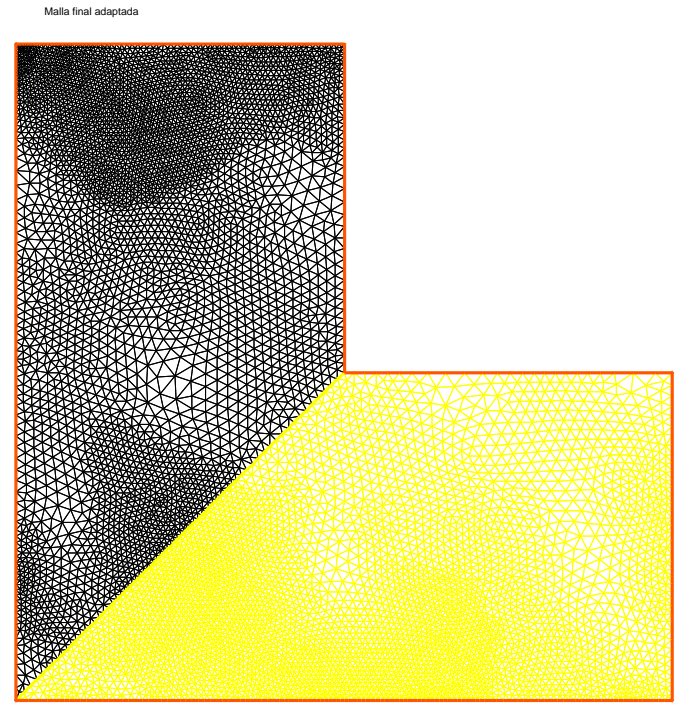

(b) Final grid

Figure 9: Triangulations of iterated solutions in adaptivity strategy 


\begin{tabular}{|c||c|c|c|c|c|c|}
\hline \multicolumn{7}{|c|}{ Comparison of $H_{00}^{1 / 2}(\Gamma)$ versus $L^{2}(\Gamma)$ penalties. } \\
\hline \hline Penalty & $\varepsilon_{\text {opt }}$ & $\eta_{h}^{P}$ & $\eta_{h}^{D}$ & Triangles & Iterations & CPU time \\
\hline$H_{00}^{1 / 2}(\Gamma)$ & 0.0387 & 0.00180 & 0.00136 & 8148 & 471 & $287.7 \mathrm{~s}$ \\
\hline$L^{2}(\Gamma)$ & 0.0035 & 0.00180 & 0.00134 & 8230 & 56 & $41.7 \mathrm{~s}$ \\
\hline \hline
\end{tabular}

Table 1.

Also, we have tested the improvement of computational time due to the adaptive strategy with respect to the single iterating Schwarz procedure. To do it we have run the Schwarz procedure, using the optimized grids and the optimal penalty parameters. We have obtained the results presented in Table 2 . We remark for both penalties a large gain of CPU time due to the adaptivity strategy, with a ratio larger than 4 in both cases.

\begin{tabular}{|c||c|c|}
\hline \multicolumn{3}{|c|}{ Schwarz iteration procedure with optimized data } \\
\hline \hline Penalty & $\varepsilon_{\text {opt }}$ & CPU time \\
\hline$H_{00}^{1 / 2}(\Gamma)$ & 0.0387 & $1158.6 \mathrm{~s}$ \\
\hline$L^{2}(\Gamma)$ & 0.0035 & $266.5 \mathrm{~s}$ \\
\hline \hline
\end{tabular}

Table 2.

\section{Penalty parameter optimization}

We have performed a final test, where only the penalty parameter is optimized for a fixed grid. As stated in Section 8, this is achieved by skipping the grid adaptation step (Step 2 ) in the adaptation algorithm. We have used a non-structured grid with 3092 triangles, corresponding to $h=1 / 64$. The procedure stops when

$$
\eta_{h}^{n+1, P} \leq \eta_{h}^{D}
$$

as in this case the discretization error is fixed, and the purpose is to decrease the penalty error below this value.

Table 3 displays the results obtained. Again, the optimal $\varepsilon$ for $L^{2}(\Gamma)$ penalty is close to the square of the optimal one for $H_{00}^{1 / 2}(\Gamma)$ penalty. Both penalties take a similar number of iterations, but the $H_{00}^{1 / 2}(\Gamma)$ one is neatly more costly.

All the results presented in this subsection are qualitatively the same for the square test. We do not present them for brevity.

\begin{tabular}{|c||c|c|c|}
\hline \multicolumn{4}{|c|}{ Penalty parameter optimization for fixed grid. } \\
\hline \hline Penalty & $\varepsilon_{\text {opt }}$ & Iterations & CPU time \\
\hline$H_{00}^{1 / 2}(\Gamma)$ & 0.0503 & 165 & $126.3 \mathrm{~s}$ \\
\hline$L^{2}(\Gamma)$ & 0.0029 & 157 & $99.0 \mathrm{~s}$ \\
\hline \hline
\end{tabular}

Table 3. 


\section{Appendix}

This appendix is devoted to the rather technical proof of Lemma 1.

Proof of (35) and (36). If some element $K \in \mathcal{T}_{i h}$ does not intersect $\Gamma$, then $\mathcal{C}_{i h}^{*} v_{i}=\mathcal{C}_{i h} v_{i}$ on $K$. Also, if some edge $e$ of some element of $\mathcal{T}_{i h}$ does not intersect $\Gamma$, then $\mathcal{C}_{i h}^{*} v_{i}=\mathcal{C}_{i h} v_{i}$ on $e$. Thus, estimates (35) and (36) are standard properties of Clément operator (Cf. [4], Théorème IX.3.7).

Proof of (37) and (38). We write the triangle inequality

$$
\left\|v-\mathcal{C}_{i h} v_{i}\right\|_{L^{2}(K)} \leq\left\|v_{i}-\mathcal{C}_{i h}^{*} v_{i}\right\|_{L^{2}(K)}+\left\|\mathcal{C}_{i h}^{*} v_{i}-\mathcal{C}_{i h} v_{i}\right\|_{L^{2}(K)} .
$$

The estimates (Cf. [4], Corollaire IX.3.10)

$$
\left\|v_{i}-\mathcal{C}_{i h}^{*} v_{i}\right\|_{L^{2}(K)} \leq C h_{K}\left|v_{i}\right|_{H^{1}\left(\Delta_{K}\right)}, \quad\left\|v_{i}-\mathcal{C}_{i h}^{*} v_{i}\right\|_{L^{2}(e)} \leq C h_{e}^{1 / 2}\left|v_{i}\right|_{H^{1}\left(\Delta_{K}\right)}, \forall e \in \mathcal{E}_{K}
$$

imply

$$
\left(\sum_{K \in \mathcal{T}_{i h}^{\Gamma}} h_{K}^{-2}\left\|v_{i}-\mathcal{C}_{i h}^{*} v_{i}\right\|_{L^{2}(K)}^{2}+\sum_{K \in \mathcal{T}_{i h}^{\Gamma}} \sum_{e \in \mathcal{E}_{K}} h_{e}^{-1}\left\|v_{i}-\mathcal{C}_{i h}^{*} v_{i}\right\|_{L^{2}(e)}^{2}\right)^{\frac{1}{2}} \leq C\left|v_{i}\right|_{H^{1}\left(\Omega_{i}\right)} .
$$

We consider now the quantity $\left\|\mathcal{C}_{i h}^{*} v_{i}-\mathcal{C}_{i h} v_{i}\right\|_{L^{2}(K)}$, and note that the support of $\mathcal{C}_{i h}^{*} v_{i}-\mathcal{C}_{i h} v_{i}$ is included in the union of the elements $K$ of $\mathcal{T}_{i h}$ that intersect $\Gamma$. On such a $K$, we have

$$
\mathcal{C}_{i h}^{*} v_{i}-\mathcal{C}_{i h} v_{i}=\sum_{a \in \mathcal{V}^{\Gamma} \cap K}\left(\left(\pi_{a} v_{i}\right)(a)-\left(\pi_{i h}^{\Gamma} v_{i}\right)(a)\right) \varphi_{a}
$$

Denote by $e_{a}$ a side or a face containing $a$ and included in $\Gamma$. Then,

$$
\left\|\mathcal{C}_{i h}^{*} v_{i}-\mathcal{C}_{i h} v_{i}\right\|_{L^{2}(K)} \leq c h_{K}^{\frac{d}{2}} \max _{a \in \mathcal{V} \Gamma K}\left\|\pi_{a} v_{i}-\pi_{i h}^{\Gamma} v_{i}\right\|_{L^{\infty}\left(e_{a}\right)} .
$$

We next use the inverse inequality

$$
\left\|\pi_{a} v_{i}-\pi_{i h}^{\Gamma} v_{i}\right\|_{L^{\infty}\left(e_{a}\right)} \leq C h_{K}^{-\frac{d-1}{2}}\left\|\pi_{a} v_{i}-\pi_{i h}^{\Gamma} v_{i}\right\|_{L^{2}\left(e_{a}\right)} .
$$

We deduce

$$
\sum_{K \in \mathcal{T}_{i h}^{\Gamma}} h_{K}^{-2}\left\|\mathcal{C}_{i h}^{*} v_{i}-\mathcal{C}_{i h} v_{i}\right\|_{L^{2}(K)}^{2} \leq C h_{m}^{-1}\left(\sum_{a \in \mathcal{V}^{\Gamma}}\left\|v_{i}-\pi_{a} v_{i}\right\|_{L^{2}\left(e_{a}\right)}^{2}+\left\|v_{i}-\pi_{i h}^{\Gamma} v_{i}\right\|_{L^{2}(\Gamma)}^{2}\right) .
$$

Consider now a side or face $e$ of some element $K$ of $\mathcal{T}_{i h}^{\Gamma}$. In this case,

$$
\left\|\mathcal{C}_{i h}^{*} v_{i}-\mathcal{C}_{i h} v_{i}\right\|_{L^{2}(e)} \leq C h_{e}^{\frac{d-1}{2}} \max _{a \in \mathcal{V}^{\Gamma} \cap K}\left\|\pi_{a} v_{i}-\pi_{i h}^{\Gamma} v_{i}\right\|_{L^{\infty}\left(e_{a}\right)}
$$


We now deduce

$$
\sum_{K \in \mathcal{T}_{i h}^{\Gamma}} \sum_{e \in \mathcal{E}_{K}} h_{e}^{-1}\left\|\mathcal{C}_{i h}^{*} v_{i}-\mathcal{C}_{i h} v_{i}\right\|_{L^{2}(e)}^{2} \leq C h_{m}^{-1}\left(\sum_{a \in \mathcal{V}^{\Gamma}}\left\|v_{i}-\pi_{a} v_{i}\right\|_{L^{2}\left(e_{a}\right)}^{2}+\left\|v_{i}-\pi_{i h}^{\Gamma} v_{i}\right\|_{L^{2}(\Gamma)}^{2}\right)
$$

Thus, the proofs of (37) and (38) are reduced to estimate the term

$$
\sum_{a \in \mathcal{V}^{\Gamma}}\left\|v_{i}-\pi_{a} v_{i}\right\|_{L^{2}\left(e_{a}\right)}^{2}+\left\|v_{i}-\pi_{i h}^{\Gamma} v_{i}\right\|_{L^{2}(\Gamma)}^{2} .
$$

To estimate the first summand of the right-hand side, we recall [4], Corollaire IX.3.9,

$$
\left\|v_{i}-\pi_{a} v_{i}\right\|_{L^{2}\left(e_{a}\right)} \leq C h_{K_{a}}^{1 / 2}\left\|v_{i}\right\|_{H^{1}\left(\Delta_{K_{a}}\right)},
$$

where $K_{a}$ is some element of the triangulation $\mathcal{T}_{i h}$ such that $a$ is a side or a face of $K_{a}$. Then,

$$
\sum_{a \in \mathcal{V}^{\Gamma}}\left\|v_{i}-\pi_{a} v_{i}\right\|_{L^{2}\left(e_{a}\right)}^{2} \leq C h_{M}|v|_{H^{1}\left(\Omega_{i}\right)}^{2} .
$$

The estimate of the second summand in the right-hand side of (55) is made as follows.

i) Assume that $\pi_{i h}^{\Gamma}$ is the $L^{2}(\Gamma)$ projector. As $\pi_{i h}^{\Gamma}$ is a projection,

$$
\left\|v_{i}-\pi_{i h}^{\Gamma} v_{i}\right\|_{L^{2}(\Gamma)}^{2} \leq\left\|v_{i}-\mathcal{C}_{i h}^{*} v_{i}\right\|_{L^{2}(\Gamma)}^{2} \leq C h_{M}\left|v_{i}\right|_{H^{1}\left(\Omega_{i}\right)}^{2}
$$

ii) Assume now that $\pi_{i h}^{\Gamma}$ is the $H_{00}^{1 / 2}(\Gamma)$ projector. Also, using that $\pi_{i h}^{\Gamma}$ is a projection,

$$
\left\|v_{i}-\pi_{i h}^{\Gamma} v_{i}\right\|_{L^{2}(\Gamma)} \leq\left\|v_{i}-\pi_{i h}^{\Gamma} v_{i}\right\|_{H_{00}^{1 / 2}(\Gamma)} \leq\left\|v_{i}\right\|_{H_{00}^{1 / 2}(\Gamma)} \leq C\left|v_{i}\right|_{H^{1}\left(\Omega_{i}\right)}
$$

Combining (52), (53) and (54) with (56), (57) and (58), we deduce (37) and (38) .

Q.E.D.

\section{Acknowledgements}

This paper was initiated when T. Chacón visited the Laboratoire Jacques-Louis Lions of the University of Paris VI, in the academic course 2005/06, funded by the Marie Curie European mobility programme. The research of T. Chacón, E. Chacón and D. Franco is also partially funded by Spanish DIG grant MTM2006-01275. 


\section{References}

[1] Adams, R. A., Sobolev Spaces. Pure and Applied Mathematics, Vol. 65. Academic Press, New-York, London, 1975.

[2] Ben Belgacem, F., The Mortar finite element method with Lagrange multipliers. Numer. Math., 84; pp. 173-197, 1999.

[3] Bernardi, C., Girault, V., Hecht, F., A posteriori analysis of a penalty method and application to the Stokes problem. Math. Models and Methods in Applied Sciences, 13; pp. 1599-1628, 2003.

[4] Bernardi, C., Maday, Y., Rapetti, F., Discrétisations variationnelles de problèmes aux limites elliptiques. Mathématiques \& Applications, 45. Springer-Verlag, Berlin, 2004.

[5] Casas, E., Raymond, J.-P., The stability in $W^{s, p}(\Gamma)$ spaces of $L^{2}$-projections on some convex sets. Numer. Funct. Anal. and Optimization, 27; pp. 117-137, 2006.

[6] Chacón Rebollo, T., Chacón Vera, E., A non-overlapping domain decomposition method for the Stokes equations via a penalty term on the interface. C.R. Acad. Sci. Paris, t. 334, Série I; pp. 1-16, 2002.

[7] Chacón Rebollo, T., Chacón Vera, E., Study of a non-overlapping domain decomposition method: Poisson and Stokes problems. Appl. Numer. Math., 48; pp. 169-194, 2004.

[8] Deng, Q., An analysis for a nonoverlapping domain decomposition iterative procedure. SIAM J. Sci. Comput., 18; pp. 1517-1525, 1997.

[9] Girault, V., Raviart, P.-A., Finite Element Methods for Navier-Stokes Equations. Theory and Algorithms. Springer Series in Computational Mathematics, 5. SpringerVerlag, Berlin, 1986.

[10] Hecht, F., Pironneau, O., www.freefem.org.

[11] Lions, J.-L., Parallel Stabilization of Hyperbolic and Petrowsky Systems. Fourth World Congress On Computational Mechanics, Buenos Aires (1998).

[12] Lions, J.-L., Magenes, E., Problèmes aux limites non homogènes et applications. Vol. 1. Travaux et Recherches Mathématiques, No. 17, Dunod, Paris, 1968.

[13] Lions, J.-L., Pironneau, O., Overlapping domain decomposition of evolution operators. C.R. Acad. Sci. Paris, t. 330, Série I; pp. 937-942, 2000.

[14] Otto, F. C., Lube, G., A posteriori estimates for a non-overlapping domain decomposition method. Computing, 62; pp. 27-143, 1999. 
[15] Quarteroni, A., Valli, A., Domain Decomposition Methods for Partial Differential Equations. Numerical Mathematics and Scientific Computations, Oxford Science Publications, 1999.

[16] Verfürth, R., A Review of a Posteriori Error Estimation and Adaptive MeshRefinement Techniques. Wiley, Chichester, England, 1996. 